\title{
The association between diet quality, dietary patterns and depression in adults: a systematic review
}

Shae E Quirk', Lana J Williams ${ }^{1,2}$, Adrienne O'Neil ${ }^{1,3}$, Julie A Pasco ${ }^{1,4}$, Felice N Jacka ${ }^{1,4,5}$, Siobhan Housden ${ }^{1}$, Michael Berk ${ }^{1,4,5,6}$ and Sharon L Brennan ${ }^{1,2,4,7^{*}}$

\begin{abstract}
Background: Recent evidence suggests that diet modifies key biological factors associated with the development of depression; however, associations between diet quality and depression are not fully understood. We performed a systematic review to evaluate existing evidence regarding the association between diet quality and depression.

Method: A computer-aided literature search was conducted using Medline, CINAHL, and PsycINFO, January 1965 to October 2011, and a best-evidence analysis performed.

Results: Twenty-five studies from nine countries met eligibility criteria. Our best-evidence analyses found limited evidence to support an association between traditional diets (Mediterranean or Norwegian diets) and depression. We also observed a conflicting level of evidence for associations between (i) a traditional Japanese diet and depression, (ii) a "healthy" diet and depression, (iii) a Western diet and depression, and (iv) individuals with depression and the likelihood of eating a less healthy diet.
\end{abstract}

Conclusion: To our knowledge, this is the first review to synthesize and critically analyze evidence regarding diet quality, dietary patterns and depression. Further studies are urgently required to elucidate whether a true causal association exists.

Keywords: Depression, Diet, Food habits, Adults, Systematic review

\section{Background}

Depressive disorders currently impose a significant health and economic burden in both developed and developing countries. With prevalence estimates ranging between $3.3-21.4 \%$ [1], the global burden of depression is now a major public health concern [2]. As such, the identification of modifiable risk factors for depression is an important and pressing research imperative [3].

Recent data have highlighted the importance of the contribution of modifiable lifestyle behaviors such as physical inactivity, smoking, and other lifestyle factors to the development of common mental disorders [4-6]. In addition, the relationship between nutrition and depressive disorders has become of increasing interest in recent years $[7,8]$

\footnotetext{
*Correspondence: sbrennan@unimelb.edu.au

${ }^{1}$ School of Medicine, Deakin University, Geelong, Australia

${ }^{2}$ Department of Psychiatry, The University of Melbourne, Parkville, Australia

Full list of author information is available at the end of the article
}

in both observational and clinical studies; however, much previous research has focused on the intake of individual nutrients or food groups and their association with depression, or on nutritional supplementation as a treatment strategy in depression.

In this regard, studies have identified associations between the intake of dietary nutrients such as zinc, magnesium, B-group vitamins, culinary fat (such as olive oil), and single food groups such as seafood or fish consumption and decreased risk of depression [8-11]. However, there are important limitations to studying individual nutrients in relation to disease, given the complex combinations and interactions among nutrients in an individual's daily diet. Diet is a multidimensional exposure and thus it remains difficult to attribute differential disease prevalence or symptomatology to a single nutrient or food group. Moreover, nutrient intake is associated with particular dietary patterns, which may act as

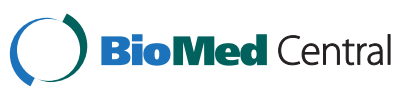


confounders in diet-disease associations. As such, dietary patterns are being increasingly examined as predictors of disease outcomes. For example, in a study of middleaged women participating in the Nurses' Health Study, a prudent dietary pattern was characterized by higher intakes of vegetables, fruit, legumes, fish, poultry and whole grains, while a western pattern was characterized by higher intakes of red and processed meats, desserts, refined grains and fried foods. These patterns were, in turn, associated with markers of systemic inflammation [12]. Moreover, a western dietary pattern has been shown to increase the risk, and a prudent dietary pattern to reduce the risk, of other inflammatory diseases, for instance, coronary heart disease in both women [13] and men [14]. Salient characteristics of diet may also be captured using a composite measure of dietary intake or dietary quality scores derived from recommended dietary guidelines. For the purpose of this review we define diet quality and dietary patterns as the quality of overall habitual dietary intake, and the pattern of overall habitual dietary intake, respectively, which is consistent with prior research $[7,15]$. Given the relatively new data available in this field, the aim of this study was to conduct the first systematic review to examine the association between overall diet quality and depression in adults.

\section{Methods}

This systematic review adheres to the guidelines addressed in the preferred reporting items for systematic reviews and meta-analyses (PRISMA) statement 2009 [16] (Additional file 1).

\section{Eligibility criteria for considering studies for this review}

Articles were eligible for inclusion if they: (i) were full-text articles; (ii) comprised cohort, case-control or crosssectional study designs; (iii) examined associations between self-reported diet quality, defined as the quality of one's overall habitual food intake ascertained by healthy eating guidelines or a priori diet quality score (rather than (1) individual nutrients, (2) individual food items or, (3) individual food groups), or dietary pattern analysis, and depression or depressive symptoms defined by either selfreport or the application of diagnostic measurement tools in adults; and (iv) comprised study samples that were population based rather than from acute settings (for example, residents at aged care facilities, in-patients at psychiatric hospitals).

\section{Criteria for excluding studies from this review}

Studies were excluded if they: (i) were published in languages other than English; (ii) utilized animal models; (iii) investigated energy intake as the primary variable of interest or outcome measure; (iv) investigated individual dietary nutrients or single dietary components as the primary variables of interest; (v) investigated malnutrition, including nutritional risk, or disordered eating; (vi) investigated parenteral nutrition as the primary variable of interest; (vii) employed qualitative methodology; (viii) were randomized controlled trials; or (ix) were dissertations. Due to differences in the diagnostic tools used to assess depression in children and/or adolescents compared to adults, we excluded studies that examined diet and depression in populations other than adults.

\section{Search strategy for identification of studies}

A computerized search strategy was implemented using Medline (largest subset of PubMed), CINAHL, and PsycINFO for citations of relevant articles, which were restricted to January 1965 to 31st October 2011. The following medical subject headings $(\mathrm{MeSH})$ were applied: "diet" OR "food habits" AND "depression" OR "depressive disorder" OR "depressive disorder, major". Keywords were applied to complete the final search strategy: "diet" OR "food habits" OR "dietary" OR "dietary patterns" OR "dietary quality" OR "western diet" or "Mediterranean diet" AND "depression (MeSH)" OR "depressive disorder" OR depressive disorder, major" OR "depression (keyword). Two reviewers confirmed the search strategy (SEQ and SLB) and one reviewer performed the computerized search (SEQ). Complete details of the search strategy can be obtained from the corresponding author.

Reference lists of relevant studies deemed eligible for inclusion were manually searched, and citations were tracked for those publishing in the field of interest (SEQ). Two reviewers (SEQ and SLB) confirmed the selection of articles based on readings of the full text article. Where the eligibility of studies was ambiguous, two reviewers held discussions to reach consensus (SEQ and SLB). Where consensus could not be achieved, a third reviewer was consulted (LJW).

\section{Methodological quality of included manuscripts}

Two reviewers (SEQ and SLB) independently assessed the quality of the studies by scoring them using an adaptation of Lievense et al.'s scoring system [17,18] (Table 1). Each of the 14 criteria items were scored as follows: positive (1), negative (0), or unclear (?) with $100 \%$ representing a maximum possible score. A third reviewer (LJW) provided a final judgment where the reviewers' agreement could not be reconciled. Studies were defined as high quality if the total quality score for all quality scores were above the mean. The optimal design was considered to be cohort studies, followed by case-control studies and, finally, cross-sectional study designs.

\section{Data analysis}

Our decision not to proceed with a meta-analysis of the data from reviewed studies was determined a priori. 
Table 1 Criteria list for the assessment of study quality, modified from Lievense et al $[15,16]$

\begin{tabular}{|c|c|c|}
\hline Item & Criteria & $\mathrm{C} / \mathrm{CC} / \mathrm{CS}^{\dagger}$ \\
\hline \multicolumn{3}{|c|}{ Study population } \\
\hline 1 & Selection at uniform point & $\mathrm{C} / \mathrm{CC} / \mathrm{CS}$ \\
\hline 2 & Cases and controls drawn from the same population & $\mathrm{CC}$ \\
\hline 3 & Participation rate $>80 \%$ for cases/cohort & $\mathrm{C} / \mathrm{CC}$ \\
\hline 4 & Participation rate $>80 \%$ for controls & $\mathrm{CC}$ \\
\hline \multicolumn{3}{|c|}{ Assessment of risk factor } \\
\hline 5 & Exposure assessment blinded & $\mathrm{C} / \mathrm{CC} / \mathrm{CS}$ \\
\hline 6 & Exposure measured identically for cases and controls & $\mathrm{CC}$ \\
\hline 7 & Exposure assessed according to validated measures & $\mathrm{C} / \mathrm{CC} / \mathrm{CS}$ \\
\hline \multicolumn{3}{|c|}{ Assessment of outcome } \\
\hline 8 & Outcome assessed identically in studied population & $\mathrm{C} / \mathrm{CC} / \mathrm{CS}$ \\
\hline 9 & Outcome reproducibly & C/CC/CS \\
\hline 10 & Outcome assessed according to validated measures & $\mathrm{C} / \mathrm{CC} / \mathrm{CS}$ \\
\hline \multicolumn{3}{|c|}{ Study design } \\
\hline 11 & Prospective design used & $\mathrm{C} / \mathrm{CC}$ \\
\hline 12 & Follow-up time $\geq 12$ months & C \\
\hline 13 & Withdrawals <20\% & C \\
\hline \multicolumn{3}{|c|}{ Analysis and data presentation } \\
\hline 14 & Appropriate analysis techniques used & $\mathrm{C} / \mathrm{CC} / \mathrm{CS}$ \\
\hline 15 & Adjusted for at least age, and gender & C/CC/CS \\
\hline
\end{tabular}

Given the current work in this field of enquiry being undertaken by the authors, our group had an appreciation of the inherent heterogeneity of these studies, largely related to measurement of diet and assessment of depression. Our "best-evidence synthesis" consisted of five levels of evidence ranging from strong evidence (1), moderate evidence (2), limited evidence (3), conflicting evidence (4), to no evidence (5), which reflected the type of study design used (Table 2).

\section{Results}

Identification and selection of the included manuscripts Utilizing Medline, CINAHL, and PsycINFO databases, the computer-assisted search generated a total of 3,826 articles, of which 646 articles were duplicates. The title and/or abstracts of the remaining 3,180 articles were screened for eligibility, of which 3,113 were excluded due to failing to meet preliminary eligibility criteria, and seven were excluded as they were printed only in languages other than English. A further 37 articles were excluded failing to meet eligibility criteria, based on a concise reading of the full articles. One further article [19] was identified when searching the reference lists of articles meeting inclusion criteria, and the e-pub of another article [20] was identified when tracking authors
Table 2 Criteria for ascertainment of evidence level for bestevidence synthesis, adapted from Lievense et al $[15,16]$

\begin{tabular}{|c|c|}
\hline $\begin{array}{l}\text { Level of } \\
\text { evidence }\end{array}$ & Criteria for inclusion in best evidence synthesis \\
\hline \multirow{2}{*}{$\begin{array}{l}\text { Strong } \\
\text { evidence }\end{array}$} & Generally consistent findings in: \\
\hline & Multiple high-quality cohort studies \\
\hline \multirow{2}{*}{$\begin{array}{l}\text { Moderate } \\
\text { evidence }\end{array}$} & Generally consistent findings in: \\
\hline & $\begin{array}{l}\text { One high-quality cohort study and }>2 \text { high } \\
\text { quality case-control studies }\end{array}$ \\
\hline \multirow{4}{*}{$\begin{array}{l}\text { Limited } \\
\text { evidence }\end{array}$} & Generally consistent findings in: \\
\hline & Single cohort study \\
\hline & One or two case-control studies or \\
\hline & Multiple cross-sectional studies \\
\hline $\begin{array}{l}\text { Conflicting } \\
\text { evidence }\end{array}$ & Inconsistent findings in $<75 \%$ of the trials \\
\hline No evidence & No studies could be found \\
\hline
\end{tabular}

publishing in the field of interest. The final number of studies to be included in the review was 25 (Figure 1).

Studies most frequently failed to meet eligibility criteria for inclusion for the following reasons (i) examined individual nutrients or supplements, rather than overall dietary quality, (ii) examined fat intake rather than overall dietary quality, (iii) examined individual food items or food groups including fish intake or meat intake, (iv) examined malnutrition or nutritional risk rather than overall dietary quality, or (v) did not measure depression or depressive symptoms adequately.

\section{Methodological quality of included manuscripts}

The two reviewers (SEQ and SLB) scored a potential 253 criteria over the 25 studies, resulting in an interrater reliability of $84 \%$. The majority of the discrepancies were resolved in one consensus meeting; however, the third reviewer (LJW) provided final judgment on $1.9 \%$ of the items.

The quality scores ranged from $55.6 \%$ [19] to $100 \%$ [7,20-23] of the maximum obtainable score for each of the study designs. Only high-quality studies (as determined by methodological assessment quality score above the mean of $87.73 \%$ ) were included in the best evidence synthesis. However, all of the reviewed studies scored above the 50\% of possible methodological quality. High levels of heterogeneity remained in the 18 studies determined as high quality $[7,8,15,20-34]$, thus statistical pooling of the extracted data was not feasible. Therefore, a best evidence synthesis was performed to assess the associations, as previously published in other fields $[17,35]$.

\section{Description of the studies}

An overview of the reviewed studies $(n=25)$ is presented in Table 3. Five of the eligible studies were a cohort design $[15,20,21,23,36]$, one was a case-control design [37] 


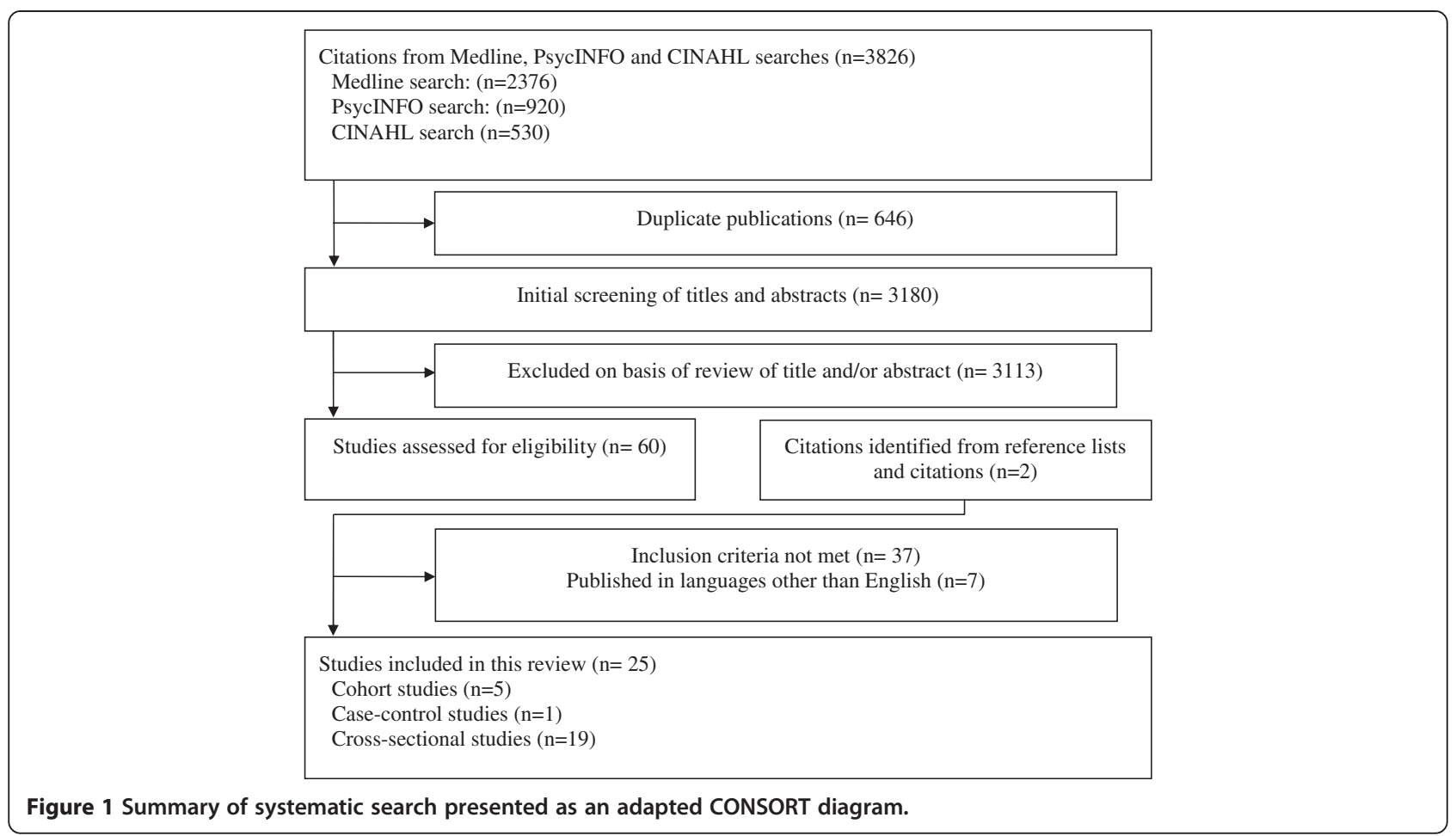

and the remaining 19 studies were cross-sectional $[7,8,19,22,24-34,38-41]$. The majority of studies were published from 2009 onwards $(n=22,84.6 \%)$, with precise publication years being; 2002 [26], 2007 [30], 2008 [25], 2009 [19,21,22,24,28,29,32,36-38], 2010 [7,33,34] and 2011 [8,15,20,23,27,31,39-41]. Eleven studies were undertaken in United States of America (USA) [19,26, 29,31-34,39-42], three were conducted in Japan [22,23,27], two were undertaken in Spain $[20,36]$. Remaining studies were conducted in the United Kingdom (UK) [21] Greece [15], Korea [37] China [30], France [25], Australia [7], Norway [8], Mediterranean Islands [28] and Europe (a combination of populations from Germany, Poland and Bulgaria) [38]. Sample sizes ranged from a cross-sectional study with 50 participants [41] to a cohort study with 10,094 participants [36] with the total number of participants examined by this review summing 53,770. Age ranges varied from 20 years in three cross-sectional studies $[7,33]$, up to 100 years in another cross-sectional study [28]. Nine of the reviewed studies examined populations comprising only females $[7,15,19,23,26,31,37,40,41]$ whilst one study comprised only of male populations [39], and the remaining 15 studies were mixed with regards to sex.

Whilst some of the studies were population-based, there were also a range of specific population groups including: a sample of white European office workers from the Whitehall II study [21], municipal office employees attending a periodic health examination [22]; university graduates and registered professionals from the Seguimiento Universidad de
Navarra/University of Navarra follow-up (SUN) cohort [20,36]; College students [30,37,38]; pregnant women $[15,23,31,41]$, and women with breast cancer [26].

A wide variety of tools, and combinations of the tools, were employed to ascertain habitual dietary intakes. These included: (i) a validated food frequency questionnaire (FFQ) $[7,8,15,20,21,26,28,36]$ (ii) independently constructed or modified versions of a validated FFQ $[19,25,30,38,40]$, (iii) validated Brief Diet History Questionnaire [22] (iv) USDA Automated Multiple Pass Method (AMPM) [32-34] (v) other dietary recall methods $[20,24,25,29,31,36,41]$, (vi) self-reported questionnaires or a single dietary habits question $[27,37,40]$ and versions of the Block FFQ [26,39]. Methods for determining diet quality also varied and included: validated Diet Quality Scores, including (vii) United States Department of Agriculture (USDA), (viii) Healthy Eating Index (HEI) or Alternative HEI [26,29,32-34,42], (ix) those based on Australian National Dietary Guidelines [7], (x) the Dietary Quality Index-Pregnancy (DQI-P) [31], (xi) Diet Health Questionnaire (DHQ) [23]; two different validated methods to measure Mediterranean diets $[28,36]$ and various other non-validated methods for assessing diet.

Similarly, a range of different methods were used to identify cases of depression, the most common being the Center for Epidemiological Studies Depression Scale (CES-D) to identify symptomatology, whilst two studies utilized psychiatric diagnostic interviews [7,33]. Another two utilized previous physician-made diagnosis of clinical depression and/ or habitual use of antidepressant medications [20,36]. 
Table 3 Study characteristics of eligible studies included in this review, grouped by study design, year of publication, and author

\begin{tabular}{|c|c|c|c|c|c|c|c|c|}
\hline \multirow[t]{2}{*}{$\begin{array}{l}\text { Author, country of } \\
\text { study, year }\end{array}$} & \multirow[t]{2}{*}{$\begin{array}{l}n=\text { subjects }(\% \\
\text { females })\end{array}$} & \multirow[t]{2}{*}{$\begin{array}{l}\text { Age, years; Mean } \\
( \pm \text { SD) or range, yr }\end{array}$} & \multirow[t]{2}{*}{ Population description } & \multicolumn{2}{|l|}{ Dietary assessment } & \multicolumn{2}{|c|}{$\begin{array}{l}\text { Depression } \\
\text { assessment }\end{array}$} & \multirow[t]{2}{*}{$\begin{array}{l}\text { Quality } \\
\text { score\% }\end{array}$} \\
\hline & & & & Tool & Type & Tool & Cut-off & \\
\hline \multicolumn{9}{|l|}{ Cohort } \\
\hline \multirow{2}{*}{$\begin{array}{l}\text { Akbaraly et al., UK, } \\
2009 \text { [21] }\end{array}$} & \multirow[t]{2}{*}{$3486(26.2)$} & \multirow[t]{2}{*}{$55.6(*), 35-55$} & \multirow{2}{*}{$\begin{array}{l}\text { White European participants in the } \\
\text { Whitehall II study with diet data at } \\
1997-9 \text {, and depression data at 2002-4 }\end{array}$} & \multirow[t]{2}{*}{ FFQ, validated, 127 items } & (i) Whole food & \multirow[t]{2}{*}{ CES-D } & \multirow[t]{2}{*}{$>15$} & \multirow[t]{2}{*}{100} \\
\hline & & & & & (ii) Processed food & & & \\
\hline $\begin{array}{l}\text { Sanchez-Villegas et al., } \\
\text { Spain, } 2009 \text { [36] }\end{array}$ & $\begin{array}{l}\text { 10,094 (\% in } \\
\text { categories of } \\
\text { adherence to Med. } \\
\text { diet; 0-2: } 59.93 \text { 3: } \\
\text { 61.4 4: 58.0 5: } 57.4 \\
\text { 6-9: 56.0) }\end{array}$ & $\begin{array}{l}\text { Age in categories of } \\
\text { adherence to Med. diet; } \\
0-2: 33.3(9.8) 3: 35.7 \\
\text { (10.7) 4: 36.8 (11.3) 5: } \\
38.0(11.6) 6-9: 41.3 \\
(12.1)\end{array}$ & $\begin{array}{l}\text { SUN Spanish cohort of former students } \\
\text { of University of Navarra, registered } \\
\text { professionals from some Spanish } \\
\text { provinces and other university } \\
\text { graduates }\end{array}$ & FFQ, validated, 136 items & Mediterranean diet & $\begin{array}{l}\text { Self- } \\
\text { reported } \\
\text { question }\end{array}$ & - & 83.3 \\
\hline \multirow{2}{*}{$\begin{array}{l}\text { Chatzi et al., Greece, } \\
2011 \text { [15] }\end{array}$} & \multirow[t]{2}{*}{$529(100)$} & \multirow[t]{2}{*}{ * } & \multirow{2}{*}{$\begin{array}{l}\text { Prospective mother-child cohort, } \\
\text { recruitment mid-pregnancy, follow up } \\
8-10 \text { weeks post-partum }\end{array}$} & \multirow{2}{*}{$\begin{array}{l}\text { FFQ, validated for this particular } \\
\text { cohort, } 250 \text { items }\end{array}$} & (i) Western pattern & \multirow[t]{2}{*}{ EPDS } & \multirow[t]{2}{*}{$\geq 13$} & \multirow[t]{2}{*}{91.7} \\
\hline & & & & & (ii) Healthy pattern & & & \\
\hline \multirow{3}{*}{$\begin{array}{l}\text { Okubu et al., Japan, } \\
2011 \text { [23] }\end{array}$} & \multirow[t]{3}{*}{$865(100)$} & \multirow[t]{3}{*}{$29.9(4.0)$} & \multirow{3}{*}{$\begin{array}{l}\text { Pregnant females enrolled in the Osaka } \\
\text { Maternal and Child Health Study, } \\
\text { recruited 2001-3, follow up 2- } \\
9 \text { months post-partum }\end{array}$} & \multirow[t]{3}{*}{ DHQ, validated, 145 items } & (i) Healthy diet & \multirow[t]{3}{*}{ EPDS } & \multirow[t]{3}{*}{$\geq 9$} & \multirow[t]{3}{*}{100} \\
\hline & & & & & (ii) Western diet & & & \\
\hline & & & & & (iii) Japanese diet & & & \\
\hline \multirow{2}{*}{$\begin{array}{l}\text { Sanchez-Villegas et al., } \\
\text { Spain, } 2011 \text { [20] }\end{array}$} & $8,964\left(^{*}\right)$ & * & SUN Spanish cohort of former students & FFQ, validated, 136 items, $2 \times 24$ hour & (i) Fast food & & - & 100 \\
\hline & & & $\begin{array}{l}\text { professionals from some Spanish } \\
\text { provinces and other university } \\
\text { graduates }\end{array}$ & & $\begin{array}{l}\text { (ii) Commercial } \\
\text { baked goods }\end{array}$ & question & & \\
\hline Case-control & & & & & & & & \\
\hline Park et al., Korea, 2010 & $130(100)$ & Cases: $20.6(0.2)$ & Korean female College students & Independently constructed self- & (i) Dietary pattern & CES-D & $\geq 16$ & 84.6 \\
\hline & & Control: 20.5 (0.2) & 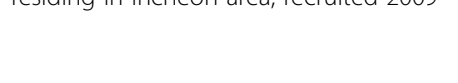 & 16 items & $\begin{array}{l}\text { beans more than } \\
\text { twice a day }\end{array}$ & & & \\
\hline & & & & & $\begin{array}{l}\text { (ii) Total dietary } \\
\text { habits score }\end{array}$ & & & \\
\hline Cross-sectional & & & & & & & & \\
\hline $\begin{array}{l}\text { Tangney et al., USA, } \\
2002 \text { [26] }\end{array}$ & $117(100)$ & $61.5\left(^{*}\right)$ & $\begin{array}{l}\text { Female breast cancer patients of urban } \\
\text { teaching hospital, cancer diagnosis 0.5- } \\
5 \text { years prior to } 1997\end{array}$ & $\begin{array}{l}\text { HHHQ transcribed to modified Block } \\
\text { FFQ, HEI }\end{array}$ & $\begin{array}{l}\text { Diet quality } \\
\text { ascertained by HEl } \\
\text { score }\end{array}$ & CES-D & $\geq 16$ & 88.9 \\
\hline $\begin{array}{l}\text { Liu et al., China, } 2007 \\
\text { [30] }\end{array}$ & $2,579(42.1)$ & $20.4(*)$ & $\begin{array}{l}\text { College students over } 7 \text { cities in China, } \\
\text { recruited 2003-4 }\end{array}$ & $\begin{array}{l}\text { Independently constructed FFQ } \\
\text { specifically for study }\end{array}$ & $\begin{array}{l}\text { (i) Ready to eat } \\
\text { food }\end{array}$ & $\begin{array}{l}\text { CES-D, } \\
\text { adapted }\end{array}$ & - & 88.9 \\
\hline & & & & & (ii) Snack food & items & & \\
\hline & & & & & (iii) Fast food & & & \\
\hline & $1,724(62.5)$ & $65+$ & & FFQ, 24 hour diet recall & & & - & 88.9 \\
\hline
\end{tabular}


Table 3 Study characteristics of eligible studies included in this review, grouped by study design, year of publication, and author (Continued)

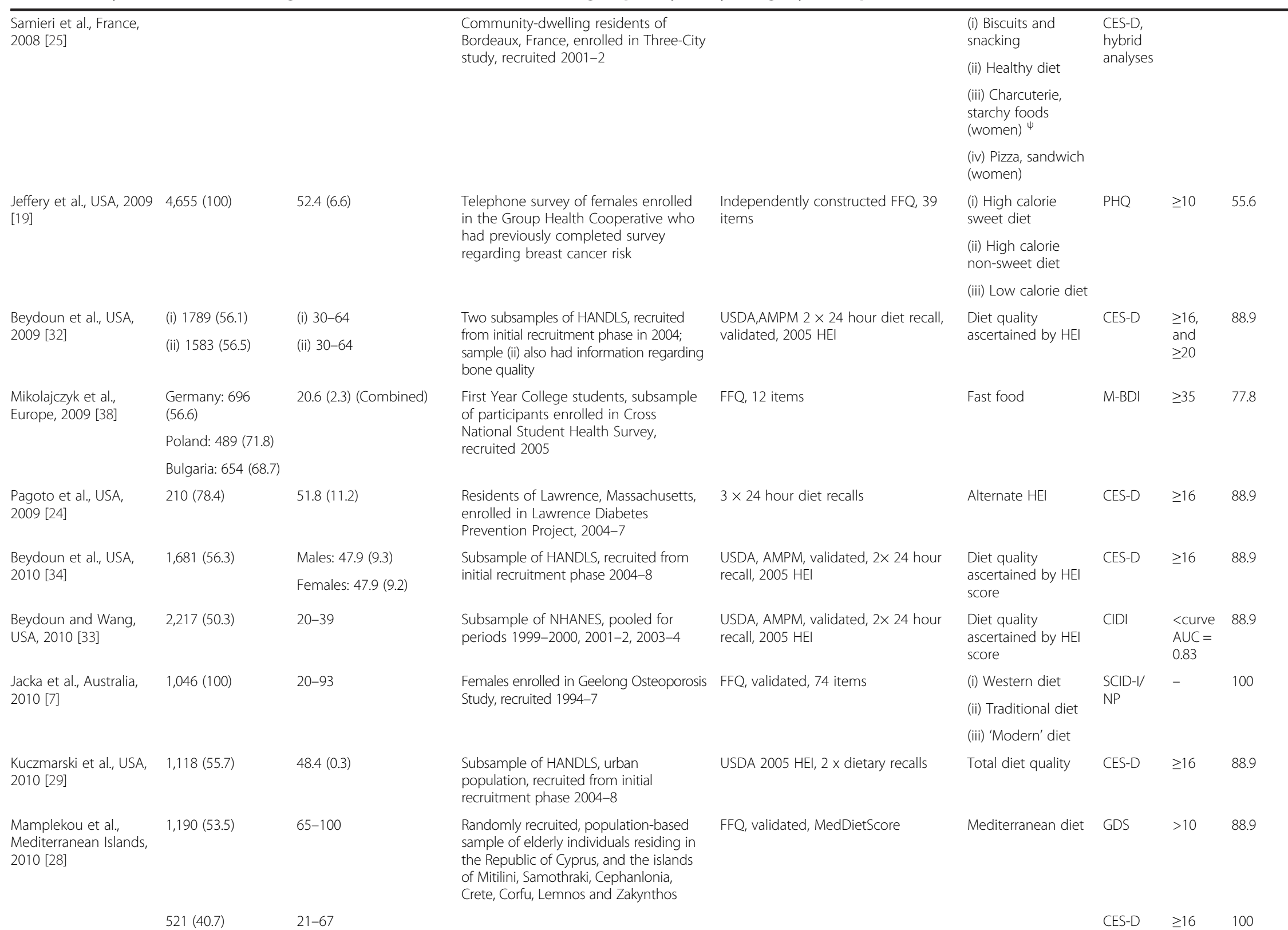

First Year College students, subsample

of participants enrolled in Cross

recruited 2005

Residents of Lawrence, Massachusetts,

nrolled in Lawrence Diabetes

Subsample of HANDLS, recruited from 
Table 3 Study characteristics of eligible studies included in this review, grouped by study design, year of publication, and author (Continued)

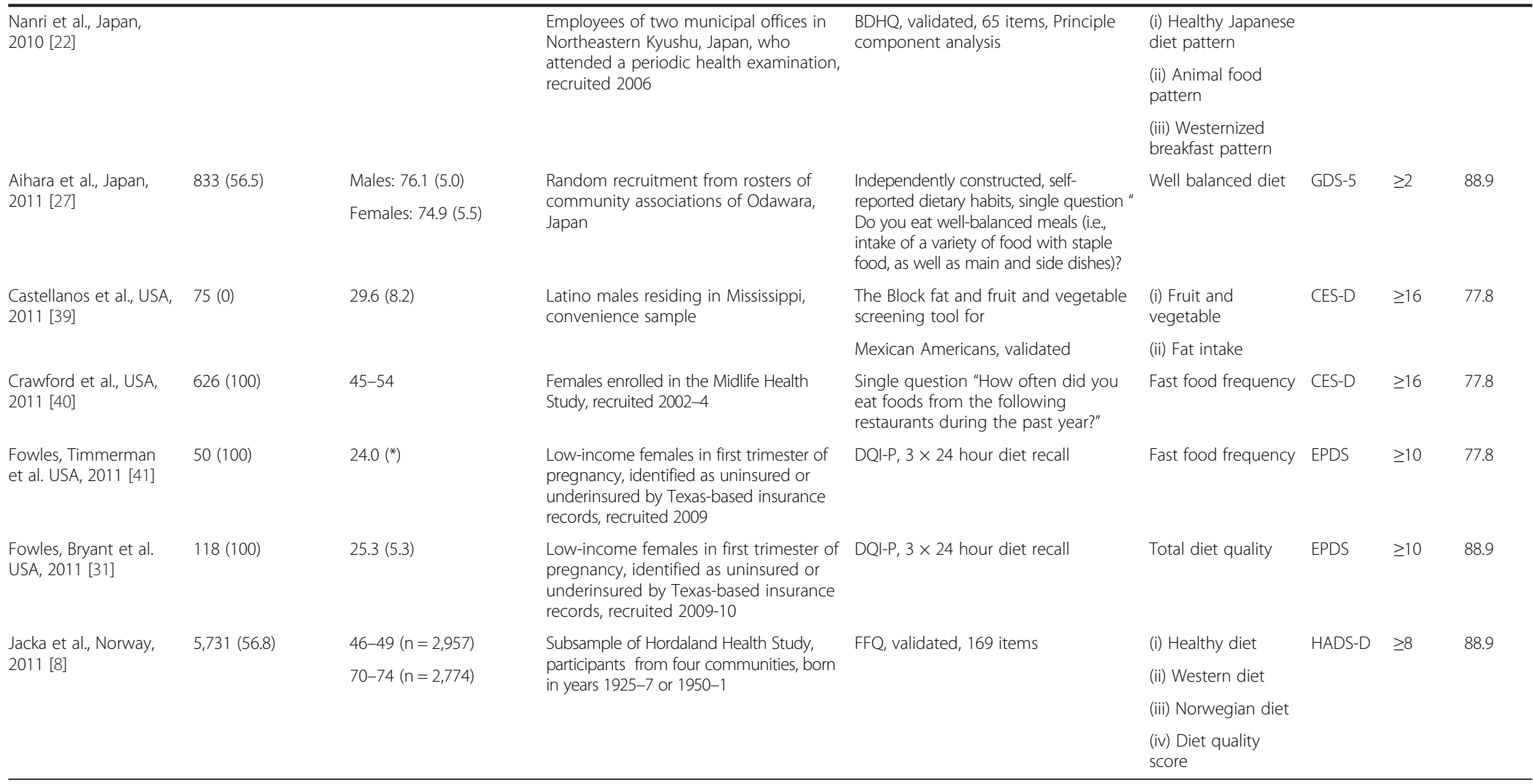

\section{* Data not provided.}

Abbreviations: FFQ Food Frequency Questionnaire, USDA United States Department of Agriculture, AMPM Automated Multiple Pass Method, HEI Healthy Eating Index, CES-D Centre for Epidemiological Studies Depression, GDS Geriatric Depression Scale, EPDS Edinburgh Postnatal Depression Scale, DHQ Diet History Questionnaire, BDHQ Brief Dietary History Questionnaire, PHQ Patient Health Questionnaire, CIDI Composite International Diagnostic Interview (Version 2.1)

SCID-I/NP Structured Clinical Interview for DSM-IV-TR Research Version, Non-Patient Edition, HADS-D Hospital Anxiety and Depression Scale for depression,

$M-B D I$ Modified Beck Depression Inventory, HANDLS Healthy Aging in Neighborhoods of Diversity across the Life Span, SUN Seguimiento Universidad de Navarra, DQI-P Dietary Quality Index-Pregnancy.

${ }^{\Psi}$ The analysis undertaken for male participants by Samieri et al. [25] was based on a food pattern of meat consumption and thus ineligible for inclusion. 
Table 4 Summary of associations between traditional dietary patterns and depression, presented by year of publication, and author

\begin{tabular}{|c|c|c|c|c|c|}
\hline Author, country, year & Type of diet & Adjusted for confounders & $\begin{array}{l}\text { Results }(\mathrm{G}=\text { group, } \\
\mathrm{T}=\text { tertile, } \mathrm{C}=\text { category, } \\
\mathrm{Q}=\text { quartile, }\end{array}$ & $p$ for trend & Summary of associations \\
\hline \multicolumn{6}{|l|}{ Cohort } \\
\hline \multirow{5}{*}{$\begin{array}{l}\text { Sanchez-Villegas et al., Spain, } \\
2009 \text { [36] }\end{array}$} & \multirow[t]{5}{*}{ Mediterranean } & \multirow{5}{*}{$\begin{array}{l}\text { Age, sex, smoking, BMl, physical activity, energy intake, } \\
\text { employment }\end{array}$} & C1: Referent & \multirow[t]{5}{*}{$<0.001$} & \multirow{5}{*}{$\begin{array}{l}\text { Increased adherence to } \\
\text { Mediterranean diet associated } \\
\text { with reduced odds of self- } \\
\text { reported depression }\end{array}$} \\
\hline & & & C2: $0.74(0.57,0.98)$ & & \\
\hline & & & C3: $0.66(0.50,0.86)$ & & \\
\hline & & & C4: $0.49(0.36,0.67)$ & & \\
\hline & & & C5: $0.58(0.44,0.77)$ & & \\
\hline \multirow{5}{*}{$\begin{array}{l}\text { Sanchez-Villegas et al., Spain, } \\
2009 \text { [36] }\end{array}$} & \multirow[t]{5}{*}{ Mediterranean } & \multirow{5}{*}{$\begin{array}{l}\text { Age, sex, smoking, BMI, physical activity, energy intake, } \\
\text { employment, excluding participants with early } \\
\text { depression }\end{array}$} & C1: Referent & \multirow[t]{5}{*}{$<0.001$} & \multirow{5}{*}{$\begin{array}{l}\text { Increased adherence to } \\
\text { Mediterranean diet associated } \\
\text { with reduced odds of self- } \\
\text { reported depression }\end{array}$} \\
\hline & & & C2: $0.73(0.50,1.06)$ & & \\
\hline & & & C3: $0.56(0.38,0.83)$ & & \\
\hline & & & C4: $0.42(0.27,0.66)$ & & \\
\hline & & & C5: $0.50(0.33,0.74)$ & & \\
\hline \multirow{5}{*}{$\begin{array}{l}\text { Sanchez-Villegas et al., Spain, } \\
2009 \text { [36] }\end{array}$} & \multirow[t]{5}{*}{ Mediterranean } & \multirow{5}{*}{$\begin{array}{l}\text { Age, sex, smoking, BMI, physical activity, energy intake, } \\
\text { employment, excluding participants using } \\
\text { antidepressant medication during follow up without } \\
\text { physician diagnosis }\end{array}$} & C1: Referent & \multirow[t]{5}{*}{0.007} & \multirow{5}{*}{$\begin{array}{l}\text { Increased adherence to } \\
\text { Mediterranean diet associated } \\
\text { with reduced odds of self- } \\
\text { reported depression }\end{array}$} \\
\hline & & & C2: $0.79(0.57,1.09)$ & & \\
\hline & & & C3: $0.67(0.48,0.93)$ & & \\
\hline & & & C4: $0.56(0.39,0.80)$ & & \\
\hline & & & C5: $0.69(0.50,0.96)$ & & \\
\hline \multirow[t]{4}{*}{ Okubu et al., Japan, 2011 [23] } & \multirow[t]{4}{*}{ Japanese } & \multirow{4}{*}{$\begin{array}{l}\text { Age, gestation, parity, smoking, change in diet in } \\
\text { preceding month, family structure, occupation, family } \\
\text { income, education, season, BMl, time of delivery, } \\
\text { medical problems during pregnancy, sex and birth } \\
\text { weight of baby }\end{array}$} & Q1: Referent & \multirow[t]{4}{*}{0.59} & \multirow[t]{4}{*}{ No association } \\
\hline & & & Q2: $0.56(0.30,1.02)$ & & \\
\hline & & & Q3: $1.14(0.66,1.96)$ & & \\
\hline & & & Q4: $0.96(0.56,1.64)$ & & \\
\hline \multicolumn{6}{|l|}{ Cross-sectional } \\
\hline \multirow{2}{*}{$\begin{array}{l}\text { Mamplekou, Mediterranean } \\
\text { Islands, } 2010[28]\end{array}$} & \multirow[t]{2}{*}{ Mediterranean } & \multirow{2}{*}{$\begin{array}{l}\text { Age, sex, BMl, living alone, financial status, physical } \\
\text { activity, smoking, co-morbidities, education, alcohol, } \\
\text { retired, urban/rural area }\end{array}$} & G1: 1.00 (ref) & \multirow[t]{2}{*}{$N S^{*}$} & \multirow[t]{2}{*}{ No association } \\
\hline & & & G2: $1.03(0.98-1.09)$ & & \\
\hline \multirow{3}{*}{$\begin{array}{l}\text { Nanri et al., Japan, } \\
2010 \text { [22] }\end{array}$} & \multirow[t]{3}{*}{ Japanese } & \multirow[t]{3}{*}{ Age, sex, workplace } & T1: Referent & \multirow[t]{3}{*}{$<0.001$} & \multirow{3}{*}{$\begin{array}{l}\text { Increased adherence to Japanese } \\
\text { diet associated with reduced } \\
\text { odds of depressive symptoms }\end{array}$} \\
\hline & & & T2: $0.90(0.57,1.41)$ & & \\
\hline & & & T3: $0.39(0.23,0.67)$ & & \\
\hline Nanri et al., Japan, & Japanese & Age, sex, workplace, marital status, BMI, job position, & T1: Referent & 0.006 & Increased adherence to Japanese \\
\hline & & $\begin{array}{l}\text { physical activity, smoking, co-morbidities, total energy } \\
\text { intake }\end{array}$ & T2: $0.99(0.62,1.59)$ & & $\begin{array}{l}\text { diet associated with reduced } \\
\text { odds of depressive symptoms }\end{array}$ \\
\hline
\end{tabular}


Table 4 Summary of associations between traditional dietary patterns and depression, presented by year of publication, and author (Continued)

\begin{tabular}{|c|c|c|c|c|c|}
\hline \multirow{6}{*}{$\begin{array}{l}\text { Jacka et al., Norway, } \\
2011 \text { [8] }\end{array}$} & \multirow[t]{6}{*}{ Norwegian } & \multirow{6}{*}{$\begin{array}{l}\text { Age, income, education, physical activity, smoking, } \\
\text { alcohol, energy consumption }\end{array}$} & Males: & \multirow{4}{*}{0.02} & \multirow{3}{*}{$\begin{array}{l}\text { Increased adherence to } \\
\text { Norwegian diet associated with } \\
\text { reduced odds of depressive } \\
\text { symptoms for males }\end{array}$} \\
\hline & & & C1: Referent & & \\
\hline & & & $C 2: 0.77(0.61,0.96)$ & & \\
\hline & & & Females: & & No association for females \\
\hline & & & C1: Referent & & \\
\hline & & & C2: $0.99(0.76,1.29)$ & 0.51 & \\
\hline
\end{tabular}

* Data not provided, NS not significant.

Results presented as Odds Ratio (OR) or Hazards Ratio (HR) and $(95 \% \mathrm{Cl})$, except where indicated by superscripts: ${ }^{\dagger}$ beta regression coefficients $( \pm \mathrm{SE})$, or ${ }^{a}$ mean $( \pm \mathrm{SE})$. 
Table 5 Summary of associations between a healthy dietary pattern and depression, presented by year of publication

\begin{tabular}{|c|c|c|c|c|c|}
\hline $\begin{array}{l}\text { Author, country, } \\
\text { year }\end{array}$ & Type of diet & Adjusted for confounders & $\begin{array}{l}\text { Results }(T=\text { tertile, } Q= \\
\text { quartile, } C=\text { category })\end{array}$ & $p$ for trend & Summary of associations \\
\hline \multicolumn{6}{|l|}{ Cohort } \\
\hline \multirow{3}{*}{$\begin{array}{l}\text { Akbaraly et al., UK, } \\
2009 \text { [21] }\end{array}$} & \multirow{3}{*}{$\begin{array}{l}\text { Whole food } \\
\text { dietary pattern }\end{array}$} & \multirow[t]{3}{*}{ Age, gender, energy intake } & T1: Referent & & \multirow{3}{*}{$\begin{array}{l}\text { Increased adherence to whole food } \\
\text { diet associated with reduced odds of } \\
\text { depressive symptoms }\end{array}$} \\
\hline & & & T2: $0.62(0.48,0.79)$ & 0.0002 & \\
\hline & & & T3: $0.64(0.49,0.83)$ & 0.001 & \\
\hline \multirow{3}{*}{$\begin{array}{l}\text { Akbaraly et al., UK, } \\
2009[21]\end{array}$} & \multirow{3}{*}{$\begin{array}{l}\text { Whole food } \\
\text { dietary pattern }\end{array}$} & \multirow{3}{*}{$\begin{array}{l}\text { Age, gender, energy intake, marital status, } \\
\text { employment, education, physical activity, } \\
\text { smoking }\end{array}$} & T1: Referent & & \multirow{3}{*}{$\begin{array}{l}\text { Increased adherence to whole food } \\
\text { diet associated with reduced odds of } \\
\text { depressive symptoms }\end{array}$} \\
\hline & & & T2: $0.68(0.52,0.89)$ & 0.004 & \\
\hline & & & T3: $0.74(0.56,0.98)$ & 0.03 & \\
\hline \multirow{3}{*}{$\begin{array}{l}\text { Akbaraly et al., UK, } \\
2009 \text { [21] }\end{array}$} & \multirow{3}{*}{$\begin{array}{l}\text { Whole food } \\
\text { dietary pattern }\end{array}$} & \multirow{3}{*}{$\begin{array}{l}\text { Age, gender, energy intake, marital status, } \\
\text { employment, education, physical activity, } \\
\text { smoking, co-morbidities, use of anti-depressant } \\
\text { drugs, cognitive functioning }\end{array}$} & T1: Referent & & \multirow{3}{*}{$\begin{array}{l}\text { Increased adherence to whole food } \\
\text { diet associated with reduced odds of } \\
\text { depressive symptoms }\end{array}$} \\
\hline & & & T2: $0.71(0.54,0.92)$ & 0.01 & \\
\hline & & & Т3: $0.74(0.56,0.99)$ & 0.04 & \\
\hline \multirow{3}{*}{$\begin{array}{l}\text { Akbaraly et al., UK, } \\
2009 \text { [21] }\end{array}$} & \multirow{3}{*}{$\begin{array}{l}\text { Whole food } \\
\text { dietary pattern }\end{array}$} & \multirow[t]{3}{*}{ Prior depression, age, gender, energy intake } & T1: Referent & & \multirow{3}{*}{$\begin{array}{l}\text { Increased adherence to whole food } \\
\text { diet associated with reduced odds of } \\
\text { depressive symptoms }\end{array}$} \\
\hline & & & T2: $0.63(0.46,0.87)$ & 0.005 & \\
\hline & & & T3: $0.66(0.47,0.92)$ & 0.01 & \\
\hline \multirow{3}{*}{$\begin{array}{l}\text { Akbaraly et al., UK, } \\
2009 \text { [21] }\end{array}$} & \multirow{3}{*}{$\begin{array}{l}\text { Whole food } \\
\text { dietary pattern }\end{array}$} & \multirow{3}{*}{$\begin{array}{l}\text { Prior depression, age, gender, energy intake, } \\
\text { marital status, employment, education, physical } \\
\text { activity, smoking }\end{array}$} & T1: Referent & & \multirow{3}{*}{$\begin{array}{l}\text { Increased adherence to whole food } \\
\text { diet associated with reduced odds of } \\
\text { depressive symptoms (non-linear) }\end{array}$} \\
\hline & & & T2: $0.70(0.50,0.96)$ & 0.03 & \\
\hline & & & T3: $0.74(0.52,1.04)$ & 0.08 & \\
\hline \multirow{3}{*}{$\begin{array}{l}\text { Akbaraly et al., UK, } \\
2009 \text { [21] }\end{array}$} & \multirow{3}{*}{$\begin{array}{l}\text { Whole food } \\
\text { dietary pattern }\end{array}$} & \multirow{3}{*}{$\begin{array}{l}\text { Prior depression, age, gender, energy intake, } \\
\text { marital status, employment, education, physical } \\
\text { activity, smoking, co-morbidities, use of anti- } \\
\text { depressant drugs, cognitive functioning }\end{array}$} & T1: Referent & & \multirow{3}{*}{$\begin{array}{l}\text { Increased adherence to whole food } \\
\text { diet associated with reduced odds of } \\
\text { depressive symptoms (non-linear) }\end{array}$} \\
\hline & & & T2: $0.68(0.50,0.94)$ & 0.02 & \\
\hline & & & Т3: $0.73(0.51,1.02)$ & 0.07 & \\
\hline \multirow{4}{*}{$\begin{array}{l}\text { Chatzi et al., Greece, } \\
2011 \text { [15] }\end{array}$} & \multirow[t]{4}{*}{ Healthy diet } & \multirow{4}{*}{$\begin{array}{l}\text { Age, education, parity, house tenure, depression } \\
\text { during previous pregnancies, total energy intake } \\
\text { during pregnancy }\end{array}$} & (Outcome: EPDS) & 0.02 & \multirow{4}{*}{$\begin{array}{l}\text { Increased adherence to healthy diet } \\
\text { associated with lower mean } \\
\text { depressive symptom scores }\end{array}$} \\
\hline & & & T1: Referent & & \\
\hline & & & T2:-1.13 $(-2.25,0.00)$ & & \\
\hline & & & T3:-1.75 $(-3.22,-0.28)$ & & \\
\hline \multirow{4}{*}{$\begin{array}{l}\text { Chatzi et al., Greece, } \\
2011 \text { [15] }\end{array}$} & Healthy diet & Age, education, parity, house tenure, depression & (Outcome: symptoms) & 0.04 & Increased adherence to healthy diet \\
\hline & & $\begin{array}{l}\text { during previous pregnancies, total energy intake } \\
\text { during pregnancy }\end{array}$ & T1: Referent & & $\begin{array}{l}\text { associated with lower mean } \\
\text { depressive symptom scores }\end{array}$ \\
\hline & & & T2: $0.52(0.30,0.92)$ & & \\
\hline & & & T3: $0.51(0.25,1.05)$ & & \\
\hline Okubu et al., Japan, & Healthy diet & Age, gestation, parity, smoking, change in diet & Q1: Referent & 0.72 & No association \\
\hline & & $\begin{array}{l}\text { in preceding month, family structure, } \\
\text { occupation, family income, education, season, }\end{array}$ & Q2: $0.82(0.46,1.47)$ & & \\
\hline & & BMI, time of delivery, medical problems during & Q3: $1.49(0.86,2.60)$ & & \\
\hline & & pre & Q4: $0.94(0.52,1.69)$ & & \\
\hline
\end{tabular}


Table 5 Summary of associations between a healthy dietary pattern and depression, presented by year of publication (Continued)

\begin{tabular}{|c|c|c|c|c|c|}
\hline \multirow[t]{2}{*}{$\begin{array}{l}\text { Park et al., Korea, } 2010 \\
\text { [37] }\end{array}$} & Total diet quality & Matched for age, sex & Cases: $47.2 \pm 0.9$ & $<0.01$ & $\begin{array}{l}\text { Increased adherence to healthier } \\
\text { total diet associated with lower } \\
\text { mean depressive symptom scores }\end{array}$ \\
\hline & & & Controls: $51.3 \pm 0.9^{a}$ & & \\
\hline \multirow[t]{2}{*}{$\begin{array}{l}\text { Park et al., Korea, } 2010 \\
\text { [37] }\end{array}$} & $\begin{array}{l}\text { Meat, fish, eggs, } \\
\text { beans }<\text { twice per } \\
\text { day }\end{array}$ & Matched for age, sex & Cases: $2.9 \pm 0.1$ & $<0.05$ & $\begin{array}{l}\text { Increased adherence to diet based } \\
\text { on meat, fish, eggs, and bean } \\
\text { associated with lower mean } \\
\text { depressive symptom scores }\end{array}$ \\
\hline & & & Controls: $3.3 \pm 0.1^{a}$ & & \\
\hline \multicolumn{6}{|l|}{ Cross-sectional } \\
\hline $\begin{array}{l}\text { Tangney et al., USA, } \\
2002 \text { [26] }\end{array}$ & Healthy & $\begin{array}{l}\text { Age, BMI, tumor characteristics (stage, node, } \\
\text { estrogen receptor), time since breast cancer } \\
\text { diagnoses }\end{array}$ & * & 0.0003 & $\begin{array}{l}\text { Increased adherence to healthy diet } \\
\text { associated with lower mean } \\
\text { depressive symptom scores }\end{array}$ \\
\hline \multirow[t]{2}{*}{$\begin{array}{l}\text { Samieri et al., France, } \\
2008 \text { [25] }\end{array}$} & Healthy & Age, education, income, marital status & $\underset{t}{\text { Males: }-0.12(-0.31,0.07)}$ & 0.21 & No association \\
\hline & & & $\begin{array}{l}\text { Females: }-0.16(-0.33 \\
0.007)^{\dagger}\end{array}$ & 0.06 & No association \\
\hline $\begin{array}{l}\text { Jeffery et al., USA, } \\
2009[19]\end{array}$ & Low calorie & $\mathrm{BMI}$, energy intake & $-0.027\left({ }^{*}\right)^{\dagger}$ & $<0.001$ & $\begin{array}{l}\text { Increased adherence to low calorie } \\
\text { diet associated with reduced odds of } \\
\text { depressive symptoms }\end{array}$ \\
\hline \multirow{2}{*}{$\begin{array}{l}\text { Beydoun et al., USA, } \\
2010[34]\end{array}$} & \multirow[t]{2}{*}{ Healthy overall } & \multirow{2}{*}{$\begin{array}{l}\text { Age, ethnicity, marital status, education, poverty } \\
\text { status, smoking, illicit drug use, BMl }\end{array}$} & Males: $-0.035(0.025)^{\dagger}$ & $N S^{*}$ & No association \\
\hline & & & Females: $-0.070(0.023)^{\dagger}$ & $<0.05$ & $\begin{array}{l}\text { Increased adherence to healthy } \\
\text { overall diet associated with reduced } \\
\text { odds of depressive symptoms for } \\
\text { females }\end{array}$ \\
\hline \multirow[t]{2}{*}{$\begin{array}{l}\text { Jacka et al., Australia, } \\
2010 \text { [7] }\end{array}$} & \multirow[t]{2}{*}{$\begin{array}{l}\text { 'Traditional' } \\
\text { (healthy) dietary } \\
\text { pattern }\end{array}$} & \multirow[t]{2}{*}{$\begin{array}{l}\text { Age, socioeconomic status, education, physical } \\
\text { activity, smoking, alcohol energy intake }\end{array}$} & C1: Referent & $<0.05$ & $\begin{array}{l}\text { Increased adherence to a traditional } \\
\text { diet (vegetables, fruit, meat, fish, } \\
\text { wholegrain foods) with reduced } \\
\text { odds of depression }\end{array}$ \\
\hline & & & C2: $0.65(0.43,0.98)$ & & \\
\hline \multirow{2}{*}{$\begin{array}{l}\text { Jacka et al., Australia, } \\
2010 \text { [7] }\end{array}$} & \multirow[t]{2}{*}{ Diet quality score } & \multirow{2}{*}{$\begin{array}{l}\text { Age, socioeconomic status, education, physical } \\
\text { activity, smoking, alcohol, energy intake }\end{array}$} & C1: Referent & NS* & No association \\
\hline & & & C2: $0.85(0.65,1.13)$ & & \\
\hline \multirow{2}{*}{$\begin{array}{l}\text { Jacka et al., Australia, } \\
2010 \text { [7] }\end{array}$} & \multirow{2}{*}{$\begin{array}{l}\text { 'Modern' dietary } \\
\text { pattern }\end{array}$} & \multirow{2}{*}{$\begin{array}{l}\text { Age, socioeconomic status, education, physical } \\
\text { activity, smoking, alcohol energy intake }\end{array}$} & C1: Referent & NS* & No association \\
\hline & & & C2: $1.29(0.96,1.73)$ & & \\
\hline $\begin{array}{l}\text { Kuczmarski et al., USA, } \\
2010 \text { [29] }\end{array}$ & $\begin{array}{l}\text { Healthy diet } \\
\text { quality }\end{array}$ & Sex, education, income, race & * & $<0.0001$ & $\begin{array}{l}\text { Increased adherence to healthy diet } \\
\text { associated with reduced odds of } \\
\text { depressive symptoms }\end{array}$ \\
\hline $\begin{array}{l}\text { Aihara et al., Japan, } \\
2011 \text { [27] }\end{array}$ & $\begin{array}{l}\text { Well-balanced } \\
\text { meals }\end{array}$ & $\begin{array}{l}\text { Age, prior depression, illness, cognitive } \\
\text { difficulties, gender }\end{array}$ & Males: & $<0.05$ & $\begin{array}{l}\text { Increased adherence to eating well- } \\
\text { balanced meals associated with } \\
\text { reduced odds of depressive } \\
\text { symptoms }\end{array}$ \\
\hline
\end{tabular}


Table 5 Summary of associations between a healthy dietary pattern and depression, presented by year of publication (Continued)

\begin{tabular}{|c|c|c|c|c|c|}
\hline & & & C1: Referent & & \\
\hline & & & $C 2: 2.75(1.25,6.05)$ & & \\
\hline & & & Females: & $<0.01$ & \\
\hline & & & C1: Referent & & \\
\hline & & & $C 2: 2.37(1.27,4.43)$ & & \\
\hline $\begin{array}{l}\text { Fowles, Bryant et al., } \\
\text { USA, } 2011 \text { [31] }\end{array}$ & Total diet quality & Age, education, social support, eating habits & $-0.293\left(*^{*}\right)^{\dagger}$ & $<0.01$ & $\begin{array}{l}\text { Healthier total diet quality associated } \\
\text { with lower mean depressive } \\
\text { symptoms }\end{array}$ \\
\hline \multirow{6}{*}{$\begin{array}{l}\text { Jacka et al., Norway, } \\
2011[8]\end{array}$} & \multirow{6}{*}{$\begin{array}{l}\text { Healthy dietary } \\
\text { pattern }\end{array}$} & \multirow{6}{*}{$\begin{array}{l}\text { Age, income, education, physical activity, } \\
\text { smoking, alcohol, energy consumption }\end{array}$} & Males: & & \\
\hline & & & C1: Referent & & \\
\hline & & & C2: $1.02(0.87,1.19)$ & 0.92 & No association \\
\hline & & & Females: & & \\
\hline & & & C1: Referent & & \\
\hline & & & C2: $0.68(0.57,0.87)$ & $<0.001$ & $\begin{array}{l}\text { Increased adherence to healthy diet } \\
\text { associated with reduced odds of } \\
\text { depressive symptoms for females }\end{array}$ \\
\hline \multirow[t]{2}{*}{$\begin{array}{l}\text { Jacka et al., Norway, } \\
2011 \text { [8] }\end{array}$} & \multirow[t]{2}{*}{ Diet quality score } & \multirow[t]{2}{*}{$\begin{array}{l}\text { Age, income, education, physical activity, } \\
\text { smoking, alcohol, energy consumption }\end{array}$} & $\begin{array}{l}\text { Males: OR }(95 \% \mathrm{CI}) \text { per SD } \\
\text { increase: } 0.83(0.70,0.99)\end{array}$ & 0.034 & \multirow{2}{*}{$\begin{array}{l}\text { Increased adherence to healthy } \\
\text { (total) diet associated with reduced } \\
\text { odds of depressive symptoms for } \\
\text { males and females }\end{array}$} \\
\hline & & & $\begin{array}{l}\text { Females: OR ( } 95 \% \mathrm{Cl}) \text { per } \\
\text { SD increase: } 0.71(0.59 \\
0.84)\end{array}$ & $<0.001$ & \\
\hline
\end{tabular}
regression coefficients $( \pm \mathrm{SE})$, or ${ }^{a}$ mean $( \pm \mathrm{SE})$. 
Table 6 Summary of associations between Western/unhealthy dietary intakes and depression, presented by year of publication, and author

\begin{tabular}{|c|c|c|c|c|c|}
\hline $\begin{array}{l}\text { Author, country, } \\
\text { year }\end{array}$ & Type of diet & Adjusted for confounders & $\begin{array}{l}\text { Results }(C=\text { category, } \\
T=\text { tertile, } Q=\text { quartile })\end{array}$ & $p$ for trend & Summary of associations \\
\hline \multicolumn{6}{|l|}{ Cohort } \\
\hline \multirow{3}{*}{$\begin{array}{l}\text { Akbaraly et al., UK, } \\
2009 \text { [21] }\end{array}$} & \multirow[t]{3}{*}{ Processed food dietary pattern } & \multirow[t]{3}{*}{ Age, gender, energy intake } & T1: Referent & & \multirow{3}{*}{$\begin{array}{l}\text { Increased consumption of } \\
\text { processed foods associated with } \\
\text { increased odds of depressive } \\
\text { symptoms }\end{array}$} \\
\hline & & & T2: $1.28(0.97,1.69)$ & 0.08 & \\
\hline & & & T3: $1.75(1.25,2.45)$ & 0.001 & \\
\hline \multirow{3}{*}{$\begin{array}{l}\text { Akbaraly et al., UK, } \\
2009 \text { [21] }\end{array}$} & \multirow[t]{3}{*}{ Processed food dietary pattern } & \multirow{3}{*}{$\begin{array}{l}\text { Age, gender, energy intake, marital status, } \\
\text { employment, education, physical activity, smoking }\end{array}$} & T1: Referent & & \multirow{3}{*}{$\begin{array}{l}\text { Increased consumption of } \\
\text { processed foods associated with } \\
\text { increased odds of depressive } \\
\text { symptoms }\end{array}$} \\
\hline & & & T2: $1.22(0.92,1.62)$ & 0.17 & \\
\hline & & & T3: $1.58(1.12,2.23)$ & 0.009 & \\
\hline \multirow{3}{*}{$\begin{array}{l}\text { Akbaraly et al., UK, } \\
2009[21]\end{array}$} & \multirow[t]{3}{*}{ Processed food dietary pattern } & \multirow{3}{*}{$\begin{array}{l}\text { Age, gender, energy intake, marital status, } \\
\text { employment, education, physical activity, smoking, } \\
\text { co-morbidities, use of anti-depressant drugs, } \\
\text { cognitive functioning }\end{array}$} & T1: Referent & & \multirow{3}{*}{$\begin{array}{l}\text { Increased consumption of } \\
\text { processed foods associated with } \\
\text { increased odds of depressive } \\
\text { symptoms }\end{array}$} \\
\hline & & & T2: $1.22(0.92,1.62)$ & 0.17 & \\
\hline & & & T3: $1.58(1.11,2.23)$ & 0.01 & \\
\hline \multirow{3}{*}{$\begin{array}{l}\text { Akbaraly et al., UK, } \\
2009 \text { [21] }\end{array}$} & \multirow[t]{3}{*}{ Processed food dietary pattern } & \multirow[t]{3}{*}{ Prior depression, age, gender, energy intake } & T1: Referent & & \multirow{3}{*}{$\begin{array}{l}\text { Increased consumption of } \\
\text { processed foods associated with } \\
\text { increased odds of depressive } \\
\text { symptoms }\end{array}$} \\
\hline & & & T2: $1.44(1.02,2.02)$ & 0.04 & \\
\hline & & & T3: $1.83(1.20,2.79)$ & 0.004 & \\
\hline \multirow{3}{*}{$\begin{array}{l}\text { Akbaraly et al., UK, } \\
2009 \text { [21] }\end{array}$} & \multirow[t]{3}{*}{ Processed food dietary pattern } & \multirow{3}{*}{$\begin{array}{l}\text { Prior depression, age, gender, energy intake, marital } \\
\text { status, employment, education, physical activity, } \\
\text { smoking }\end{array}$} & T1: Referent & & \multirow{3}{*}{$\begin{array}{l}\text { Increased consumption of } \\
\text { processed foods associated with } \\
\text { increased odds of depressive } \\
\text { symptoms }\end{array}$} \\
\hline & & & T2: $1.41(1.00,2.00)$ & 0.05 & \\
\hline & & & T3: $1.76(1.14,2.70)$ & 0.01 & \\
\hline \multirow{3}{*}{$\begin{array}{l}\text { Akbaraly et al., UK, } \\
2009 \text { [21] }\end{array}$} & \multirow[t]{3}{*}{ Processed food dietary pattern } & \multirow{3}{*}{$\begin{array}{l}\text { Prior depression, age, gender, energy intake, marital } \\
\text { status, employment, education, physical activity, } \\
\text { smoking, co-morbidities, use of anti-depressant } \\
\text { drugs, cognitive functioning }\end{array}$} & T1: Referent & & \multirow{3}{*}{$\begin{array}{l}\text { Increased consumption of } \\
\text { processed foods associated with } \\
\text { increased odds of depressive } \\
\text { symptoms }\end{array}$} \\
\hline & & & T2: $1.38(0.98,1.95)$ & 0.06 & \\
\hline & & & T3: $1.69(1.10,2.60)$ & 0.02 & \\
\hline \multirow{4}{*}{$\begin{array}{l}\text { Chatzi et al., Greece, } \\
2011 \text { [15] }\end{array}$} & \multirow[t]{4}{*}{ Western diet } & \multirow{4}{*}{$\begin{array}{l}\text { Age, education, parity, house tenure, depression } \\
\text { during previous pregnancies, total energy intake } \\
\text { during pregnancy }\end{array}$} & (Outcome: EPDS) & 0.07 & \multirow[t]{4}{*}{ No association } \\
\hline & & & T1: Referent & & \\
\hline & & & T2: $0.96(-0.17,2.00)$ & & \\
\hline & & & T3: $1.32(-0.19,2.76)$ & & \\
\hline \multirow{4}{*}{$\begin{array}{l}\text { Chatzi et al., Greece, } \\
2011 \text { [15] }\end{array}$} & Western diet & Age, education, parity, house tenure, depression & (Outcome: symptoms) & 0.70 & No association \\
\hline & & during pregnancy & T1: Referent & & \\
\hline & & & T2: $1.10(0.63,1.93)$ & & \\
\hline & & & T3: $1.14(0.58,2.26)$ & & \\
\hline Okubu et al., Japan, & Western diet & Age, gestation, parity, smoking, change in diet in & Q1: Referent & 0.36 & No association \\
\hline & & $\begin{array}{l}\text { preceding month, family structure, occupation, } \\
\text { family income, education, season, BMI, time of }\end{array}$ & Q2: $0.52(0.30,0.93)$ & & \\
\hline & & delivery, medical problems during pregnancy, sex & Q3: $0.71(0.41,1.20)$ & & \\
\hline & & welgnt & Q4: $0.73(0.42,1.24)$ & & \\
\hline & Fast food consumption & Age, sex & Q1: Referent & 0.01 & \\
\hline
\end{tabular}


Table 6 Summary of associations between Western/unhealthy dietary intakes and depression, presented by year of publication, and author (Continued)

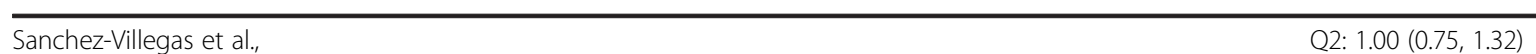

Spain, $2011[20]$

Sanchez-Villegas et al., Fast food consumption Spain, 2011 [20]

Sanchez-Villegas et al., Fast food consumption Spain, $2011[20]$

Sanchez-Villegas et al., Fast food consumption Spain, $2011[20]$

Sanchez-Villegas et al., Commercial baked goods Spain, 2011 [20] consumption

Sanchez-Villegas et al., Commercial baked goods Spain, 2011 [20] consumption

Sanchez-Villegas et al., Commercial baked goods Spain, 2011 [20] consumption
Age, sex, smoking, physical activity, total energy intake, BMl

Q3: $0.98(0.73,1.32)$

Q4: $1.04(0.78,1.39)$

Q5: $1.45(1.09,1.92)$

Q1: Referent

Q2: $0.99(0.74,1.32)$

Q3: $0.97(0.72,1.30)$

Q4: $1.02(0.76,1.38)$

Q5: $1.40(1.05,1.86)$

Q1: Referent

Age, sex, smoking, physical activity, total energy intake, BMI, consumption of commercial baked goods

Q2: $0.99(0.74,1.32)$

Q3: $0.95(0.70,1.27)$

Q4: $1.00(0.75,1.35)$

Q5: $1.36(1.02,1.81)$

Age, sex, smoking, physical activity, total energy intake, BMI, consumption of healthy food items

Q1: Referent

Q2: $0.99(0.74,1.32)$

Q3: $0.98(0.73,1.32)$

Q4: $1.03(0.76,1.39)$

Q5: $1.37(1.02,1.83)$

Q1: Referent

Q2: $1.38(1.03,1.85)$

Q3: $1.33(0.99,1.79)$

Q4: $1.10(0.81,1.49)$

Q5: $1.40(1.05,1.87)$

Q1: Referent

Q2: $1.44(1.06,1.95)$

Q3: $1.40(1.01,1.94)$

Q4: $1.15(0.82,1.61)$

Q5: $1.43(1.06,1.93)$

Q1: Referent

Q2: $1.41(1.04,1.93)$

Q3: $1.37(0.99,1.90)$

Q4: $1.12(0.79,1.57)$

Q5: $1.38(1.02,1.87)$

Age, sex smoking, physical activity, total energy intake, BMI, consumption of fast food
Increased consumption of fast odds of self-reported depression

0.01

Increased consumption of fast foods associated with increased odds of self-reported depression
0.02

Increased consumption of fast foods associated with increased odds of self-reported depression

No association 


$\begin{array}{ll}\text { Sanchez-Villegas et al., } & \text { Commercial baked goods } \\ \text { Spain, } 2011 \text { [20] } & \text { consumption }\end{array}$

Spain, 2011 [20]
Age, sex, smoking, physical activity, total energy intake, BMI, consumption of healthy food items

Cross-sectional

Liu et al., China, $2007 \quad$ Fast food

[30]

Liu et al., China, 2007 Ready to eat food

[30]

Liu et al., China, 2007 Snack food

[30]

Samieri et al., France, Females: Pizza, sandwich

2008 [25]

Samieri et al., France, Biscuits and snacking

2008 [25]

Samieri et al.,France, Females: Charcuterie, starchy 2008 [25]

Jeffery et al., USA,

2009 [19]

Jeffery et al., USA,

2009 [19]

Mikolajczyk et al.,

Europe, 2009 [38]

Fast food

High calorie sweet diet

High calorie non-sweet diet 2010 [7]

Nanri et al., Japan, 2010 [22]
BMI, energy intake

Country

Age, socioeconomic status, education, physical activity, smoking, alcohol, energy intake

Sex, current year of College study, city, weight, smoking, alcohol

Sex, current year of College study, city, weight, smoking, alcohol

Sex, current year of College study, city, weight,

Age, education, income, marital status

Age, education, income, marital status

Age, education, income, marital status

BMI, energy intake

T2: $0.99(0.63,1.57)$

T3: $1.21(0.75,1.95)$

Age, sex, workplace smoking, alcohol

Q1: Referen

Q2: $1.42(1.05,1.93)$

Q3: $1.36(0.98,1.89)$

Q4: $1.13(0.80,1.58)$

Q5: $1.37(1.01,1.85)$

Females $0.34(*)^{\dagger}$

C1: Referent

C2: $1.52(0.96,2.41)$

T1: Referent

0.32

No association

\section{T1: Referent}

2: $0.89(0.23,3.46)$

T1: Referent

$0.96(0.77,1.18)$

T3: $0.70(0.57,0.86)$

Females: $0.21(-0.11,0.53)^{\dagger} \quad 0.19$

Males: $-0.06(-0.35,0.23)^{\dagger} \quad 0.70$

Females: $0.13(-0.07,0.33)^{\dagger} \quad 0.19$

Females: $-0.15(-0.32,0.02)^{\dagger} 0.07$

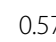

NS*

0.43

No association
$<0.01$

$<0.0$

0.02

$S^{*}$

$N S^{*}<0.0001$ food associated with reduced odds of depressive symptoms

Decreased consumption of ready to eat food associated with reduced odds of depressive symptoms

Decreased consumption of snack food associated with reduced odds of depressive symptoms

No association

No association

No association

Decreased consumption of high calorie sweet foods associated with lower mean depressive

symptom scores

Decreased consumption of high calorie non-sweet foods associated with lower mean depressive symptom scores

Increased consumption of fast foods associated with greater mean depressive symptom scores for men

\section{No association}

No association

No association 
Table 6 Summary of associations between Western/unhealthy dietary intakes and depression, presented by year of publication, and author (Continued)

\begin{tabular}{|c|c|c|c|c|c|}
\hline \multirow{3}{*}{$\begin{array}{l}\text { Nanri et al., Japan, } \\
2010 \text { [22] }\end{array}$} & \multirow[t]{3}{*}{ Westernized breakfast pattern } & \multirow{3}{*}{$\begin{array}{l}\text { Age, sex, workplace, marital status, BMl, job position, } \\
\text { physical activity, smoking, co morbidities, total } \\
\text { energy intake }\end{array}$} & T1: Referent & \multirow[t]{3}{*}{0.34} & \multirow[t]{3}{*}{ No association } \\
\hline & & & T2: $1.02(0.64,1.64)$ & & \\
\hline & & & T3: $1.27(0.77,2.10)$ & & \\
\hline \multirow{3}{*}{$\begin{array}{l}\text { Nanri et al., Japan, } \\
2010 \text { [22] }\end{array}$} & \multirow[t]{3}{*}{ Animal food pattern } & \multirow[t]{3}{*}{ Age, sex, workplace } & T1: Referent & \multirow[t]{3}{*}{0.94} & \multirow[t]{3}{*}{ No association } \\
\hline & & & T2: $1.43(0.92,2.23)$ & & \\
\hline & & & T3: $0.99(0.63,1.55)$ & & \\
\hline \multirow{3}{*}{$\begin{array}{l}\text { Nanri et al., Japan, } \\
2010 \text { [22] }\end{array}$} & \multirow[t]{3}{*}{ Animal food pattern } & \multirow{3}{*}{$\begin{array}{l}\text { Age, sex, workplace, marital status, BMl, job position, } \\
\text { physical activity, smoking, co morbidities, total } \\
\text { energy intake }\end{array}$} & T1: Referent & \multirow[t]{3}{*}{0.91} & \multirow[t]{3}{*}{ No association } \\
\hline & & & T2: $1.47(0.93,2.32)$ & & \\
\hline & & & T3: $0.97(0.61,1.55)$ & & \\
\hline $\begin{array}{l}\text { Fowles, Timmerman } \\
\text { et al., USA, } 2011 \text { [41] }\end{array}$ & Fast food frequency & Matched for age, sex & $T-2.5(-6.45,0.71)$ & $<0.05$ & $\begin{array}{l}\text { Increased consumption of fast } \\
\text { foods associated with higher } \\
\text { mean depressive symptom } \\
\text { scores }\end{array}$ \\
\hline \multirow{6}{*}{$\begin{array}{l}\text { Jacka et al., Norway, } \\
2011 \text { [8] }\end{array}$} & \multirow[t]{6}{*}{ Western dietary pattern } & \multirow{6}{*}{$\begin{array}{l}\text { Age, income, education, physical activity, smoking, } \\
\text { alcohol, energy consumption }\end{array}$} & Males: & & \\
\hline & & & C1: Referent & & \\
\hline & & & $C 2: 0.87(0.68,1.11)$ & 0.25 & No association \\
\hline & & & Females: & & \\
\hline & & & C1: Referent & & \\
\hline & & & $\mathrm{C} 2 ; 1.25(0.93,1.68)$ & 0.27 & No association \\
\hline
\end{tabular}

Results presented as Odds Ratio (OR) or Hazards Ratio (HR) and (95\% Cl), except where indicated by superscripts: ${ }^{\dagger}$ beta regression coefficients $( \pm \mathrm{SE}$ ),

or ${ }^{a}$ mean $( \pm \mathrm{SE})$.

* Data not provided. ${ }^{\psi}$ The analysis undertaken for male participants by Samieri et al. [25] was based on a food pattern of meat consumption and thus ineligible for inclusion. 
Table 7 Summary of associations between depression (exposure of interest) and diet, presented by year of publication

\begin{tabular}{|c|c|c|c|c|c|}
\hline $\begin{array}{l}\text { Author, country, } \\
\text { year }\end{array}$ & $\begin{array}{l}\text { Type of diet } \\
\text { (outcome) }\end{array}$ & Adjusted for confounders & Results & $p$ value & Summary of associations \\
\hline \multicolumn{6}{|l|}{ Cross-sectional } \\
\hline $\begin{array}{l}\text { Pagoto et al., USA, } \\
2009 \text { [24] }\end{array}$ & Healthy Eating & Age, sex, smoking & $-2.03(0.60)^{\dagger}$ & 0.001 & $\begin{array}{l}\text { Depressive symptoms associated with } \\
\text { reduced likelihood of healthy eating }\end{array}$ \\
\hline \multirow{8}{*}{$\begin{array}{l}\text { Beydoun et al., USA, } \\
2009 \text { [32] }\end{array}$} & \multirow[t]{8}{*}{ Healthy Eating } & \multirow{8}{*}{$\begin{array}{l}\text { Age, poverty status, education, } \\
\text { marital status, smoking }\end{array}$} & White males: & & \\
\hline & & & $\left(\right.$ CES-D)-0.25 $(0.08)^{\dagger}$ & $<0.05$ & $\begin{array}{l}\text { Depressive symptoms associated with } \\
\text { reduced likelihood of healthy eating }\end{array}$ \\
\hline & & & $(C E S-D \geq 16)-3.44(1.62)^{\dagger}$ & $N S^{*}$ & $\begin{array}{l}\text { Depressive symptoms associated with } \\
\text { reduced likelihood of healthy eating }\end{array}$ \\
\hline & & & $(C E S-D \geq 20)-2.82(1.99)^{\dagger}$ & $<0.05$ & No association \\
\hline & & & White females: & & \\
\hline & & & $\left(\right.$ CES-D)-0.19 $(0.07)^{\dagger}$ & $<0.05$ & $\begin{array}{l}\text { Depressive symptoms associated with } \\
\text { reduced likelihood of healthy eating }\end{array}$ \\
\hline & & & $(C E S-D \geq 16)-3.45(1.26)^{\dagger}$ & & $\begin{array}{l}\text { Depressive symptoms associated with } \\
\text { reduced likelihood of healthy eating }\end{array}$ \\
\hline & & & $(C E S-D \geq 20)-3.93(1.46)^{\dagger}$ & & $\begin{array}{l}\text { Depressive symptoms associated with } \\
\text { reduced likelihood of healthy eating }\end{array}$ \\
\hline \multirow{8}{*}{$\begin{array}{l}\text { Beydoun et al., USA, } \\
2009 \text { [32] }\end{array}$} & \multirow[t]{8}{*}{ Healthy Eating } & \multirow{8}{*}{$\begin{array}{l}\text { Age, poverty status, education, } \\
\text { marital status, smoking }\end{array}$} & African American males: & & \\
\hline & & & $(C E S-D)-0.03(0.07)^{\dagger}$ & $N S^{*}$ & No association \\
\hline & & & $(C E S-D \geq 16)-0.08(1.22)^{\dagger}$ & $N S^{*}$ & No association \\
\hline & & & $(C E S-D \geq 20)-0.90(1.52)^{\dagger}$ & $N S^{*}$ & No association \\
\hline & & & African American females: & & \\
\hline & & & $(C E S-D)-0.10(0.06)^{\dagger}$ & $<0.1$ & No association \\
\hline & & & $(C E S-D \geq 16)-1.24(1.04)^{\dagger}$ & $N S^{*}$ & No association \\
\hline & & & $(C E S-D \geq 20)-1.22(1.20)^{\dagger}$ & $N S^{*}$ & No association \\
\hline \multirow{2}{*}{$\begin{array}{l}\text { Beydoun and Wang, } \\
\text { USA, } 2010 \text { [33] }\end{array}$} & \multirow[t]{2}{*}{ Healthy Eating } & \multirow{2}{*}{$\begin{array}{l}\text { Age, race/ethnicity, marital status, } \\
\text { food insecurity, education, poverty } \\
\text { income ratio }\end{array}$} & Males: $-3.29(2.12)^{\dagger}$ & $\mathrm{NS}^{*}$ & No association \\
\hline & & & Females: $-2.63(1.96)^{\dagger}$ & $N S^{*}$ & No association \\
\hline $\begin{array}{l}\text { Castellanos et al., USA, } \\
2011 \text { [39] }\end{array}$ & Fat intake & $\begin{array}{l}\text { Age, income, education, fruit/ } \\
\text { vegetable intake, time in USA }\end{array}$ & $-0.23(0.14)^{\dagger}$ & 0.12 & No association \\
\hline $\begin{array}{l}\text { Castellanos et al., USA, } \\
2011 \text { [39] }\end{array}$ & $\begin{array}{l}\text { Fruit and Vegetable } \\
\text { consumption }\end{array}$ & $\begin{array}{l}\text { Age, income, education, fat } \\
\text { consumption, time in USA }\end{array}$ & $-0.30(0.09)^{\dagger}$ & $<0.05$ & $\begin{array}{l}\text { Depressive symptoms associated with } \\
\text { reduced likelihood of fruit and vegetable } \\
\text { consumption }\end{array}$ \\
\hline \multirow[t]{2}{*}{$\begin{array}{l}\text { Crawford et al., USA, } \\
2011[40]\end{array}$} & \multirow[t]{2}{*}{$\begin{array}{l}\text { Frequency of fast } \\
\text { food consumption }\end{array}$} & \multirow{2}{*}{$\begin{array}{l}\text { Age, race, marital status, education, } \\
\text { household income, BMI, smoking, } \\
\text { physical activity, anti-depressant use }\end{array}$} & C1: Referent & $S^{*}$ & $\begin{array}{l}\text { Depressive symptoms associated with } \\
\text { greater fast food consumption }\end{array}$ \\
\hline & & & C2: $1.54(1.06,2.25)$ & & \\
\hline
\end{tabular}




\section{Study groupings}

Due to the substantial heterogeneity of the study designs, particularly in relation to measures of diet quality, subjective decisions were required regarding the way studies were grouped. As such, studies addressing dietary constructs clearly designed to assess the habitual intake of foods known to be healthy were grouped. Similarly, 'western' dietary patterns and dietary constructs assessing the intake of foods of lower dietary quality, including takeaway foods, processed foods and those with high sugar and/or fat content, were grouped. Finally, culturally specific 'traditional' dietary patterns were grouped, which included Japanese, Norwegian and Mediterranean.

\section{Findings of the studies}

Results of the 25 reviewed studies are presented in Tables 4, 5, 6 and 7, according to the exposure of interest. Where possible, results are presented in the form of odds ratio (OR) with 95\% confidence intervals $(95 \% \mathrm{CI})$, or beta coefficient and standard error (SE) or $95 \%$ CI; $p$ values are provided where available.

\section{Best evidence synthesis}

\section{Traditional diets and the risk/likelihood of depression}

One high-quality cross-sectional study [28] reported no association between a traditional Mediterranean diet and the likelihood of depressive symptoms. As such, according to the criteria for level of evidence, this is considered to be limited evidence.

Similarly, one high-quality cross-sectional study [8] reported no association between increased adherence to a traditional Norwegian diet and a reduced likelihood of depressive symptoms; again resulting in a limited level of evidence.

One high-quality cohort study [23] identified no association between a traditional Japanese diet and the likelihood of depressive symptoms. In contrast, one high-quality cross-sectional study [22] identified that greater adherence to a traditional Japanese diet was associated with a reduced likelihood of depressive symptoms. Therefore, a conflicting level of evidence exists for an association between a Japanese diet and the likelihood of depression.

\section{Healthy, low-calorie, or whole food diets, or well-balanced meals and the risk/likelihood of depression}

One high-quality cohort study [23] reported no association between a healthy diet and the likelihood of depression; findings similarly observed by two highquality cross-sectional studies $[7,25]$. Furthermore, another high-quality cross-sectional study [34] reported an association between a healthy diet and depression for females (although not for males), whilst a further study [8] reported that an association existed for males (although not for females).

In contrast, one high-quality cohort study [15] reported a significant association between adherence to a healthy diet and a reduced likelihood of depression, an association which was similarly reported by three highquality cross-sectional studies [26,27,29], and also observed in another cross-sectional study [34] (albeit in females only). Another high-quality cohort study [21] also reported a significant association between increased adherence to a whole food diet and the reduced likelihood of depression. A third high-quality cross-sectional study [7] reported a significant association between the consumption of a traditional diet, characterized by fruit, vegetables, lean meats and whole grains, and a reduced likelihood of depression.

Given that two high-quality cohort and four crosssectional studies reported a healthy diet reduced the likelihood of depression, whilst one high-quality cohort and various cross-sectional studies reported no association, we report a conflicting level of evidence exists.

\section{Western or less healthy diets and the risk/likelihood of depression}

Three high-quality cohort studies $[15,20,23]$ and four highquality cross-sectional studies $[7,8,22,25]$ provided no evidence for an association between a Western diet and the likelihood of depression or depressive symptomatology.

Two analyses from high-quality cohort studies [20,21] and one high-quality cross-sectional study [30] reported significant associations between reduced consumption of Western foods/less healthy diets and a decreased likelihood of depressive symptoms.

Given that two high-quality cohorts and one crosssectional study reported that the consumption of Western foods/ increased the likelihood of depression, whilst three high-quality cohort and four cross-sectional studies reported no association, we report a conflicting level of evidence exists.

\section{Depression as a predictor of diet quality}

In studies that examined depression as the exposure variable of interest, one high-quality cross-sectional study [24] reported a significant association between depressive symptoms and the reduced likelihood of eating a healthy diet; that association was also reported in a second high-quality cross-sectional study [32] in white males and females, however not for African American males or females. Similarly, another high-quality crosssectional study [33] found no association between depression and the likelihood of eating a less healthy diet. Given these data, a conflicting level of evidence exists for the association between depression and diet. 


\section{Discussion}

This review identified an emerging body of research that examined the association between diet quality and patterns and the likelihood of depression. Of the available literature, we found only limited evidence to support an association between traditional diets (i.e. Mediterranean diet, Norwegian diet) and depression, after applying a best evidence analysis. We also observed a conflicting level of evidence for associations between (i) a traditional Japanese diet and depression, (ii) a healthy diet and depression, (iii) a Western diet and depression, and (iv) depression and the likelihood of eating a less healthy diet.

When investigating possible reasons for these inconsistent findings overall, as well as amongst comparable studies, similar themes emerged. Notwithstanding the robust methodological quality of most of the included studies, a high level of heterogeneity was observed in relation to the measurement of diet quality, depression assessment and study samples. Particularly notable were the heterogeneous definitions of 'healthy diet' and the wide variance in the measurement of diet quality and patterns. It is acknowledged that the complexity in measurement is an inherent issue in this field of research, with high levels of measurement error attenuating observable associations, which may help explain the inconsistencies observed. Indeed, this issue precluded us from conducting a meta-analysis.

Similarly, the wide variance in the instruments used to measure depression may have obscured or diluted potentially significant associations between depression and diet quality. Despite a diagnostic interview being considered a more accurate method for classifying depression than self-report methods, only two studies employed a formal diagnostic tool to identify depression $[7,33]$ with the majority of studies capturing depressive symptomatology rather than depression per se. However, it should be acknowledged that some of the studies reviewed had a large sample size which would make the use of diagnostic interviews impractical [36].

Alternatively, variance in the key characteristics of the study populations may help explain the inconsistent findings observed. For example, where some studies used population-based cohorts, others comprised participants who may have greater susceptibility to depression, such as those with pre-diabetes [24] and cancer [26]. It is acknowledged that synthesizing data derived from different study populations increases the likelihood of bias which affects the generalizability and comparability of findings. Moreover, it is plausible that physical illnesses or other conditions such as pregnancy may act as confounders in the relationship between diet quality and depression. A final methodological consideration is the variation in statistical modeling techniques and covariates included across studies to analyze the association between diet and depression. For instance, we draw particular attention to energy intake. The inclusion of this covariate by some (e.g. [19-21] but not all [27,29] may be potentially problematic. While some of the observed associations between depression and diet remained with (e.g. [19-21]) or without [27,29] adjustment for energy consumption, there were instances where significant relationships between (western) dietary pattern and depression was explained by this variable [7], an association that is also observed with regards to anxiety [8]. In this instance, the authors hypothesized that, in the relationship between a 'western' dietary pattern and mental health, it may likely be the "absolute amount of unhealthy food consumed" more than the "quantity as a proportion of overall diet' that is of importance. Furthermore, the high correlation between energy intake and western dietary patterns may be problematic [8]. We recommend that future studies investigating the relationship between diet and depression take this into account. More specifically, we recommend that statistical analyses in this area of research employ a priori design, where covariates are explicitly identified prior to the undertaking of analyses to ensure methodological rigor.

If, in fact, a true causal association between diet quality and depression exists but is being masked by methodological shortcomings, this is of great clinical and public health significance. Not only is diet a potentially modifiable risk factor which may support population prevention strategies, but dietary improvement could provide a novel therapeutic strategy for those with existing depression. Given that significant numbers of people fail to respond to pharmacological and/or psychological treatments, this is an area of psychiatric research that warrants greater attention.

It should be noted however, that there are inherent limitations to observational studies in regards to determining causality. The majority of studies included in this review were cross-sectional. Whilst cross-sectional study designs do not provide information regarding the directionality of associations, this review is a reflection of the existing evidence base. We therefore recommend the enactment of higher quality studies that are sufficiently powered to determine causality when exploring the relationship between diet and mental health. Any association between diet quality and patterns and mental illness are likely influenced by a large number of interrelated factors. It is plausible that demographic (e.g. socio-economic position), bio-behavioral, genetic, environmental and socio-cultural factors all contribute to the demonstrated associations. Individual analyses undertaken in the studies included in our review all controlled for some key confounding factors in their analysis, most commonly, age and gender. In addition, the large majority of studies adjusted for socioeconomic variables, such as 
education and income, as well as other lifestyle behaviors that have been shown to confound the diet-depression relationship [43]. Other studies showed energy intake to contribute to the association between depression and diet [7]. Moreover, the relationship between depression and diet is plausibly bi-directional, with individuals with depression more likely to consume poorer quality diets [44]. Two of the studies [20,21] reviewed tested the 'reverse causality' hypothesis and excluded this as an explanation; however, this relationship is complex and difficult to explicate using observational data. It was beyond the scope of this study to examine the overall nutritional components of the dietary patterns and draw comparisons with single food items, however, future studies investigating these links are warranted. The authors acknowledge the variance in the definitions of dietary patterns, which may have reduced comparability of the studies included in this review. However, this issue reflects the broader and inherent complexities often confronted in this area of research. A major strength of this review is that this evidence is for the first time, synthesised and analysed to provide an epidemiological evidence base for the association between diet and depression.

It is also important to note that there is a rapidly developing evidence base supporting the role of diet in the genesis of depression in children [45] and adolescents [46-49]. Therefore, it is acknowledged that a greater number of longitudinal studies that explore the role of diet in the development of mental disorders across the lifespan are required. In the context of this review, having data from a greater number of cohort studies may have altered the findings in the best evidence synthesis. Furthermore, greater variation may exist in dietary habits and quality between, rather than within, countries; a factor that plausibly exists for adults, adolescents and children with regards to depression, as has been seen with regards to the seafood consumption in those with bipolar disorder [50].

We acknowledge that grey literature and dissertations regarding these associations may exist. Whilst the exclusion of these sources of literature may result in our review reflecting less than the existing evidential base, it was beyond the scope of this study to systematically ascertain and review grey literature and dissertations. Notwithstanding the limitations of the available literature, this review has several strengths. To our knowledge, this is the first review to systematically explore associations between diet quality and depression. Our findings provide the basis for further inquiry to determine whether a causal relationship exists between diet quality and depression. Given the relative infancy of this area of research, we suggest that the construction of a standard definition for dietary quality and patterns would enhance future work in this area of enquiry. Higher quality cohort studies using more consistent measures of diet quality or dietary patterns to ensure findings are generalisable and comparable are required; the validation of such tools would, in time, further enhance our understanding of these associations. It is acknowledged that social and cultural factors make the examination of diet as a risk factor for depression challenging, as potential relationships may vary over time and in relation to psychological symptomatology, environmental, and/or contextual factors. Therefore, in addition to quality longitudinal studies, clinical trials designed to evaluate dietary intervention as depression prevention and/or management strategy should be conducted in an attempt to clarify this complex relationship. Moreover, studies that investigate biomarkers as mediators of observed relationships between diet quality and depression may help to clarify potentially causal mechanisms.

\section{Conclusions}

In summary, this systematic review provides a critical summary of the current evidence regarding diet quality and depression, a relatively new field of enquiry. To elucidate whether true causal associations exist between diet and depression, further research is urgently required.

\section{Additional file}

\section{Additional file 1: PRISMA 2009 Checklist.}

\section{Competing interests}

Lana J Williams has received Grant/Research support from Eli Lilly, Pfizer, The University of Melbourne, Deakin University and the NHMRC. Julie A Pasco has received grant support from the NHMRC, the Geelong Region Medical Research Foundation, Barwon Health, Perpetual Trustees, the Dairy Research and Development Corporation, The University of Melbourne, the Ronald Geoffrey Arnott Foundation, ANZ Charitable Trust, Eli Lilly, the American Society for Bone and Mineral Research and Amgen (Europe) GmBH. Felice N Jacka has received Grant/Research support from the Brain and Behaviour Research Institute (formerly NARSAD), the National Health and Medical Research Council (NHMRC), Australian Rotary Health, the Geelong Medical Research Foundation, the lan Potter Foundation, Eli Lilly and The University of Melbourne and has been a paid speaker for Sanofi-Synthelabo, Janssen Cilag, Servier, Pfizer, Network Nutrition, and Eli Lilly. Michael Berk has received Grant/Research Support from the NIH, Cooperative Research Centre, Simons Autism Foundation, Cancer Council of Victoria, Stanley Medical Research Foundation, MBF, NHMRC, Beyond Blue, Geelong Medical Research Foundation, Bristol Myers Squibb, Eli Lilly, Glaxo SmithKline, Organon, Novartis, Mayne Pharma and Servier, has been a speaker for Astra Zeneca, Bristol Myers Squibb, Eli Lilly, Glaxo SmithKline, Janssen Cilag, Lundbeck, Merck, Pfizer, Sanofi Synthelabo, Servier, Solvayand Wyeth, and served as a consultant to Astra Zeneca, Bristol Myers Squibb, Eli Lilly, Glaxo SmithKline, Janssen Cilag, Lundbeck and Servier. There are no further conflicting interests/disclosures.

\section{Authors' contributions}

SEQ and FNJ conceived the study. SEQ, SLB and FNJ designed the study and interpreted the data. SEQ and SLB undertook the acquisition of the data, and took primary responsibility for writing the manuscript. All authors assisted with the interpretation of the analysis and critically revised the manuscript, and read and approved the final manuscript. 


\section{Acknowledgements}

Lana J Williams is the recipient of an Alfred Deakin Postdoctoral Fellowship. Adrienne O'Neil is supported by an NHMRC Project Grant (1021347). FN Jacka is supported by an NHMRC Early Career Fellowship (628912). Sharon L Brennan is the recipient of NHMRC Early Career Fellowship (1012472). There are no funding bodies associated with this particular research study.

\section{Author details}

${ }^{1}$ School of Medicine, Deakin University, Geelong, Australia. ${ }^{2}$ Department of Psychiatry, The University of Melbourne, Parkville, Australia. ${ }^{3}$ School of Public Health and Preventive Medicine, Monash University, Melbourne, Australia. ${ }^{4}$ Northwest Academic Centre, Department of Medicine, The University of Melbourne, Sunshine Hospital, 176 Furlong Road, St Albans, Australia. ${ }^{5}$ Orygen Youth Health Research Centre, Parkville, Australia. ${ }^{6}$ Mental Health Research Institute, Parkville, Australia. 'Australian Institute for Musculoskeletal Science, The University of Melbourne, 176 Furlong Road, St Albans, Australia.

Received: 11 February 2013 Accepted: 21 June 2013

Published: 27 June 2013

\section{References}

1. Kessler RC, Angermeyer M, Anthony JC, De Graaf R, Demyttenaere K, Gasquet I, et al: Lifetime prevalence and age-of-onset distributions of mental disorders in the world health Organization's world mental health survey initiative. World Psychiatry 2007, 6(3):168-176

2. World Health Organization Press: World Health Organization $(W H O)$ the global burden of disease, 2004 update. 20 Avenue Appia, 1211 Geneva 27, Switzerland; 2004.

3. Collins PY, Patel V, Joestl SS, March D, Insel TR, Daar AS, Anderson W, Dhansay MA, Phillips A, Shurin S, et al: Grand challenges in global mental health. Nature 2011, 475:27-30

4. Pasco JA, Williams LJ, Jacka FN, Ng F, Henry MJ, Nicholson GC, Kotowicz MA, Berk M: Tobacco smoking as a risk factor for major depressive disorder: a population-based study. Brit J Psychiat 2008, 193(4):322-326.

5. Pasco JA, Williams LJ, Jacka FN, Henry MJ, Coulson CE, Brennan SL, Leslie E, Nicholson GC, Kotowicz MA, Berk M: Habitual physical activity and the risk for depressive and anxiety disorders among older men and women. Int Psychogeriatr 2011, 23(2):292-298.

6. Sacker A, Cable N: Do adolescent leisure-time physical activities foster health and well-being in adulthood? Evidence from two British birth cohorts. Eur J Public Health 2006, 16(3):332-336.

7. Jacka FN, Pasco JA, Mykletun A, Williams LJ, Hodge AM, O'Reilly SL, Nicholson GC, Kotowicz MA, Berk M: Association of western and traditional diets with depression and anxiety in women. The Amer $J$ Psychiat 2010, 167(3):305-311.

8. Jacka FN, Mykletun A, Berk M, Bjelland I, Tell GS: The association between habitual diet quality and the common mental disorders in communitydwelling adults: the hordaland health study. Psychosom Med 2011, 73(6):483-490.

9. Tolmunen T, Hintikka J, Ruusunen A, Voutilainen S, Tanskanen A, Valkonen V-P, Viinamaki H, Kaplan GA, Salonen JT: Dietary folate and the risk of depression in Finnish middle-aged men: a prospective follow-up study. Psychother Psychosom 2004, 73(6):334-339.

10. Murakami K, Mizoue T, Sasaki S, Ohta M, Sato M, Matsushita Y, et al: Dietary intake of folate, other B vitamins, and omega-3 polyunsaturated fatty acids in relation to depressive symptoms in Japanese adults. Nutrition 2008, 24(2):140-147

11. Sanchez-Villegas A, Henriquez P, Figueiras A, Ortuno F, Lahortiga F, Martinez-Gonzalez MA: Long chain omega-3 fatty acids intake, fish consumption and mental disorders in the SUN cohort study. Europ J Nutr 2007, 46(6):337-346.

12. Lopez-Garcia E, Schulze MB, et al: Major dietary patterns are related to plasma concentrations of markers of inflammation and endothelial dysfunction. Am J Clin Nutr 2004, 80(4):1029-1035.

13. Fung $\Pi$, Willett WC, et al: Dietary patterns and the risk of coronary heart disease in women. Arch Intern Med 2001, 161(15):1857-1862.

14. Hu FB, Rimm EB, et al: Prospective study of major dietary patterns and risk of coronary heart disease. Am J Clin Nutr 2000, 72(4):912-921.

15. Chatzi L, Melaki V, Sarri K, Apostolaki I, Roumeliotaki T, Georgiou V, Vassilaki $M$, Koutis A, Bitsios P, Kogevinas M: Dietary patterns during pregnancy and the risk of postpartum depression: the mother-child 'Rhea' cohort in Crete, Greece. Public Health Nut 2011, 14(9):1663-1670.

16. Moher D, Liberati A, Tetzlaff J, Altman DG: Preferred reporting items for systematic reviews and meta-analyses: The PRISMA Statement. PLoS Med 2009, 6(7):1-6.

17. Lievense A, Bierma-Zeinstra SMA, Vergahen AP, van Baar ME, Verhaar JAN, Koes BW: Influence of obesity on the development of osteoarthritis of the hip: a systematic review. Rheumatol 2002, 41(10):1155-1162.

18. Lievense A, Bierma-Zeinstra SMA, Verhagen AP, Verhaar JAN, Koes BW: Influence of work on the development of osteoarthritis of the hip: a systematic review. J Rheumatol 2001, 28:2520-2528.

19. Jeffery RW, Linde JA, Simon GE, Ludman EJ, Rohde P, Ichikawa LE, Finch EA: Reported food choices in older women in relation to body mass index and depressive symptoms. Appetite 2009, 52:238-240.

20. Sanchez-Villegas A, Toledo E, de Irala J, Ruiz-Canela M, Pla-Vidal J, MartinezGonzalez MA: Fast-food and commercial baked goods consumption and the risk of depression. Public Health Nut 2011, 15(3):424-432.

21. Akbaraly TN, Brunner EJ, Ferrie JE, Marmot MG, Kivimaki M, Singh-Manoux A: Dietary pattern and depressive symptoms in middle age. The Brit J Psychiat 2009, 195(5):408-413

22. Nanri A, Kimura Y, Matsushita Y, Ohta M, Sato M, Mishima N, Sasaki S, Mizoue T: Dietary patterns and depressive symptoms among Japanese men and women. Eur J Clinical Nutrition 2010, 64(8):832-839.

23. Okubo H, Miyake Y, Sasaki S, Tanaka K, Murakami K, Hirota Y: Dietary patterns during pregnancy and the risk of postpartum depression in Japan: the Osaka maternal and child health study. The Brit J Nut 2011, 105(8):1251-1257.

24. Pagato SL, Ma Y, Bodenlos JS, Olendzki B, Rosal MC, Tellez T, Merriam P, Ockene IS: Association of depressive symptoms and lifestyle behaviors among Latinos at risk of type 2 diabetes. J Amer Dietetic Assoc 2009, 109(7):1246-1250.

25. Samieri C, Jutand MA, Feart C, Capuron L, Letenneur L, PBG: Dietary patterns derived by hybrid clustering method in older people: association with cognition, mood, and self-rated health. J Amer Dietetic Assoc 2008, 108(9):1461-1471

26. Tangney CC, Young JA, Murtaugh MA, Cobleigh MA, Oleske DM: Selfreported dietary habits, overall dietary quality and symptomatology of breast cancer survivors: a cross-sectional examination. Breast Cancer Res Treat 2002, 71(2):113-123.

27. Aihara Y, Minai J, Aoyama A, Shimanouchi S: Depressive symptoms and past lifestyle among Japanese elderly people. Comm Mental Health J 2011, 47(2):186-193.

28. Mamplekou E, Bountziouka V, Psaltopoulou T, Zeimbekis A, Tsakoundakis N, Papaerakleous N, Gotsis E, Metallinos G, Pounis G, Polychronopoulos E, Lionis C, Panagiotakos D: Urban environment, physical inactivity and unhealthy dietary habits correlate to depression among elderly living in eastern Mediterranean islands: the MEDIS (MEDiterranean ISlands Elderly) study. J Nutr Health Aging 2010, 14(6):449-455.

29. Kuczmarski MF, Cremer Sees A, Hotchkiss L, Cotugna N, Evans MK, Zonderman AB: Higher healthy eating index-2005 scores associated with reduced symptoms of depression in an urban population: findings from the healthy aging in Neighborhoods of diversity across the life span (HANDLS) study. J Amer Dietetic Assoc 2010, 110(3):383-389.

30. Liu C, Xie B, Chou C-P, Koprowski C, Zhou D, Palmer P, Sun P, Guo Q, Duan $L$, Sun $X$, Anderson Johnson C: Perceived stress, depression and food consumption frequency in the college students of china seven cities. Physiol Behavior 2007, 92(4):748-754.

31. Fowles ER, Bryant M, Kim S, Walker LO, Ruiz RJ, Timmerman GM, Brown A: Predictors of dietary quality in low-income pregnant women: a path analysis. Nurs Res 2011, 60(5):286-294.

32. Beydoun MA, Kuczmarski MT, Mason MA, Ling SM, Evans MK, Zonderman AB: Role of depressive symptoms in explaining socioeconomic status disparities in dietary quality and central adiposity among US adults: a structural equation modeling approach. Amer J Clinical Nutr 2009, 90(4):1084-1095.

33. Beydoun MA, Wang Y: Pathways linking socioeconomic status to obesity through depression and lifestyle factors among young US adults. J Affect Dis 2010, 123(1-3):52-63.

34. Beydoun MA, Fanelli Kuczmarski MT, Beydoun HA, Shroff MR, Mason MA Evans MK, Zonderman AB: The sex-specific role of plasma folate in mediating the association of dietary quality with depressive symptoms. The J Nut 2010, 140(2):338-347. 
35. Brennan SL, Pasco JA, Urquhart DM, Oldenburg B, Wang Y, Wluka AE: Association between socioeconomic status and bone mineral density in adults: a systematic review. Osteoporos Int 2010, 22(2):517-527.

36. Sanchez-Villegas A, Delgado-Rodriguez M, Alonso A, Schlatter J, Lahortiga F, Serra Majem L, Martinez-Gonzalez MA: Association of the Mediterranean dietary pattern with the incidence of depression: the seguimiento Universidad de Navarra/university of Navarra follow-up (SUN) cohort. Arch Gen Psychiat 2009, 66(10):1090-1098.

37. Park J-Y, You J-S, Chang K-J: Dietary taurine intake, nutrients intake, dietary habits and life stress by depression in Korean female college students: a case-control study. J Biomed Sci 2010, 17(Suppl 1):S40.

38. Mikolajczyk RT, El Ansari W, Maxwell AE: Food consumption frequency and perceived stress and depressive symptoms among students in three European countries. Nutr J 2009, 8(31):31.

39. Castellanos DC, Connell C, Lee J: Factors affecting weight gain and dietary intake in Latino males residing in Mississippi: a preliminary study. Hispanic Health Care Int 2011, 9(2):91-98.

40. Crawford GB, Khedkar A, Flaws JA, Sorkin JD, Gallicchio L: Depressive symptoms and self-reported fast-food intake in midlife women. Prev Med 2011, 52(3-4):254-257.

41. Fowles ER, Timmerman GM, Bryant M, Kim S: Eating at fast-food restaurants and dietary quality in low-income pregnant women. Western J Nursing Research 2011, 33(5):630-651.

42. Pagoto SL, Ma Y, Bodenlos JS, Olendzki B, Rosal MC, Tellez T, Merriam P, Ockene IS: Association of depressive symptoms and lifestyle behaviors among Latinos at risk of type 2 diabetes. J Amer Dietetic Assoc 2009, 109(7):1246-1250.

43. Darmon N, Drewnowski A: Does social class predict diet quality? Am J Clin Nutr 2008, 87:1107-1117.

44. Kilian R, Becker T, Kruger K, Schmid S, Frasch K: Health behaviour in psychiatric in-patients compared with a German general population sample. Acta Psychiatrica Scand 2006, 114(4):242-248.

45. Kohlboeck G, Sausenthaler S, Standl M, et al: Food intake, diet quality and behavioural problems in children: Results from the GINI-plus/LISA-plus studies. Ann Nutr Metab 2012, 60(4):247-256.

46. Jacka FN, Kremer PJ, Berk M, de Silva-Sanigorski AM, Moodie M, et al: A prospective study of diet quality and mental health in adolescents. PLoS One 2011, 6(9):e24805.

47. Weng $\Pi$, Hao JH, Qian QW, et al: Is there any relationship between dietary patterns and depression and anxiety in Chinese adolescents? Public Health Nut 2011, 25:1-10.

48. Oddy WH, Robinson M, Ambrosini GL, et al: The association between dietary patterns and mental health in early adolescence. Prev Med 2009, 23(49):39-44.

49. Jacka FN, Kremer PJ, Leslie E, et al: Associations between diet quality and depressed mood in adolescents: results from the Health Neighbourhoods study. Aust NZ J Psychiat 2010, 44(5):435-442.

50. Noaghiul S, Hibbeln JR: Cross-national comparisons of seafood consumption and rates of bipolar disorders. Am J Psychiatry 2003, 160(12):2222-2227.

doi:10.1186/1471-244X-13-175

Cite this article as: Quirk et al: The association between diet quality, dietary patterns and depression in adults: a systematic review. BMC Psychiatry 2013 13:175.

\section{Submit your next manuscript to BioMed Central and take full advantage of:}

- Convenient online submission

- Thorough peer review

- No space constraints or color figure charges

- Immediate publication on acceptance

- Inclusion in PubMed, CAS, Scopus and Google Scholar

- Research which is freely available for redistribution 